\title{
L'exaltation de la mémoire : une approche bergsonienne des expériences de mort imminente
}

Evrard, R. (2017). L'exaltation de la mémoire : une approche bergsonienne des expériences de mort imminente. Intellectica, 68, 257-289.

\section{Résumé}

En 1896, dans son essai sur la relation du corps et de l'esprit, répondant au titre de Matière et mémoire, Bergson utilise l'étrange phénomène de la vision panoramique de la vie à l'appui de sa théorie des deux mémoires. Il développe à nouveau cet exemple dans son discours de président de la Society for Psychical Research en 1913 où il situe le déclencheur de cette exaltation de la mémoire non pas dans un simple déséquilibre sensori-moteur, mais dans une brusque démobilisation de l'élan vital. L'intérêt de Bergson pour ce phénomène est replacé, d'une part, dans le contexte scientifique de son époque et, d'autre part, dans sa théorie des deux mémoires. Puis nous prolongeons l'analyse bergsonienne à partir de données anciennes ou récentes portant plus largement sur les expériences de mort imminente. La vision panoramique de la vie et d'autres phénomènes associés sont intégrés dans une " expérience de la mémoire pure » ou "conscience désincorporée», dans laquelle est observée une distanciation des relations entre l'esprit et le corps. Les phénomènes d'automatismes intelligents et les performances physiques et intellectuelles favorisant la survie - des aspects des EMI méconnus de Bergson - montrent que la conscience désincorporée se complète d'une « expérience de la perception pure » ou «conscience hyperincorporée ». Celle-ci rend compte d'un rapprochement ou focalisation dans les relations entre l'esprit et le corps, dans le but pratique de survivre lors d'une confrontation à une menace perçue comme mortelle. La combinaison des deux consciences procède donc d'une disjonction de processus habituellement intriqués, suivie d'une nouvelle jonction souvent problématique lorsque prend fin la perception de la menace vitale.

Mots-clefs : mémoire pure - expérience de mort imminente - vision panoramique de la vie - Henri Bergson - automatismes - encorporation

\section{The exaltation of memory: a Bergsonian approach of near-death experiences}

\begin{abstract}
In 1896, in his essay on the relationship between the body and the mind, entitled Matter and Memory, Bergson used the strange phenomenon of the panoramic vision of life in support of his theory of the two memories. He developed this example again in his presidential address to the Society for Psychical Research in 1913 where he located the trigger of this exaltation of the memory not merely in a simple sensory-motor imbalance, but in a sudden demobilization of the vital impulse. Bergson's interest in this phenomenon is placed, on the one hand, in the scientific context of his time and, on the other, in his theory of the two memories. Then we extend the Bergsonian analysis on the basis of old and recent data on the near-death experiences (NDE). The panoramic vision of life and other associated phenomena are integrated into an "experience of pure memory" or "disembodied consciousness," in which there is a distancing of the relations between the mind and the body. The phenomena of intelligent automatisms and the physical and intellectual performances favoring survival - aspects of NDE that Bergson
\end{abstract}


neglected - show that the disembodied consciousness is complemented by an "experience of pure perception" or "hyperembodied consciousness." It reflects a rapprochement or focus in the relationship between the mind and the body, with the practical purpose of surviving in a confrontation with a threat perceived as mortal. The combination of the two consciousness thus proceeds from a disjunction of processes usually entangled, followed by a new and often problematic junction when the perception of the vital threat ends.

Key-words: pure memory - near-death experience - paranoramic view of life - Henri Bergson - automatisms - embodiment

Renaud EVRARD, MCF-HDR, Université de Lorraine, Laboratoire Interpsy, 23 boulevard Albert 1 ${ }^{\text {er }, 54000 ~ N a n c y . ~ r e n a u d . e v r a r d @ u n i v-l o r r a i n e . f r ~}$

$* * *$

\section{Introduction}

Les expériences de mort imminente (EMI) font l'objet d'un nombre croissant de recherches depuis que le philosophe et psychiatre Raymond Moody a popularisé en 1975 le concept de near-death experiences et son acronyme NDE (Moody, 1975). Elles sont définies comme des expériences inhabituelles entraînant parfois de profonds bouleversements psychologiques qui surviennent lorsqu'un individu se trouve physiologiquement ou psychologiquement à proximité d'une menace mortelle (Holden, Greyson, \& James, 2009 ; Corman et al., 2017). L'incidence des EMI, selon des études prospectives dans quatre pays, est en moyenne de $17 \%$ lorsque mesurée avec un outil standardisé (van Lommel et al., 2001 ; Zingrone \& Alvarado, 2009).

Cependant, à la fin du XIX ${ }^{\mathrm{e}}$ siècle, ces mêmes expériences avaient déjà suscité l'intérêt de plusieurs psychologues, psychiatres, physiologistes et philosophes (Alvarado, 2011; Evrard, Lazrak, Laurent, Toutain, Le Maléfan, sous presse). Parmi eux se trouvaient le philosophe français Henri Bergson qui tenait les expériences de mort imminente parmi les faits les plus instructifs venant soutenir sa théorie des deux mémoires indépendantes, la « mémoire habitude » et la " mémoire pure ». Il le fit une première fois en 1896 dans Matière et mémoire (Bergson, 1896), livre qui aura de l'influence sur les modèles de l'esprit de son époque. Préoccupé par ce phénomène, il y est revenu à maintes reprises et en parlait avec ses amis (La Harpe, 1941, p. 361). La seconde fois fut en 1911 dans une conférence à l'Université d'Oxford sur la perception du changement (Bergson, 1934), et la troisième fois, dans son discours de 1913 lorsqu'il prit la présidence de la Society for Psychical Research (SPR) (Bergson, 1913, 1914-1915). A notre connaissance, aucune recherche n'a été entreprise pour analyser en profondeur cette possible convergence entre la théorie bergsonienne et la recherche sur les EMI, laquelle a évolué depuis les premières collectes de cas qui ont renseigné le philosophe. Un tel travail peut favoriser une meilleure compréhension de la théorie des deux mémoires et, réciproquement, apporter un éclairage négligé sur la psychologie des EMI. Selon certains auteurs (Parnia, 2017), l'EMI pourrait nous renseigner sur l'expérience cognitive du trépas, c'est-à-dire ce que nous percevons, pensons et nous remémorons lorsque nous avons l'impression d'être sur le point de mourir. Des recherches récentes sur les souvenirs d'EMI ont mis en évidence leur singularité, puisqu'ils donnent lieu à des sensations complexes et scénarisées dans des circonstances dramatiques. Ils laissent 
une empreinte qui non seulement se distingue des souvenirs d'événements imaginaires (Thonnard et al., 2013 ; Palmieri et al., 2014), mais paraissent subjectivement plus réels que la réalité (Moore \& Greyson, 2017). Certains chercheurs arguent d'une absence de théorie capable de rendre compte de la riche phénoménologie des souvenirs d'EMI (Martial et al., 2017), en ignorant totalement la contribution de Bergson (Poulet, 1960). Ceux qui mentionnent la théorie bergsonienne le font parfois d'une façon si tronquée, et sans s'appuyer sur ses propres analyses de ces vécus, qu'ils en arrivent à conclure que les données des recherches sur les EMI vont à l'encontre du modèle bergsonien (Rousseau, 2011, p.424). Cet article se donne pour objectif de rappeler, de façon synthétique, en quoi consiste la théorie bergsonienne des deux mémoires et de quelle façon elle peut véritablement être illustrée par les EMI. Même si nous ne nous donnons pour tâche ni de prouver la validité de cette théorie - qui a fait l'objet de critiques à la lumière des neurosciences contemporaines (Gallois \& Forzy, 1997) - ni d'établir la nature des EMI, nous proposerons quelques hypothèses qui permettraient de vérifier la pertinence et la fécondité du modèle bergsonien par rapport aux autres hypothèses explicatives.

\section{Mémoire panoramique et moi vif}

Bergson est un philosophe atypique qui a mis en avant l'intuition comme méthode philosophique rigoureuse (Deleuze, 1966), tout en intégrant également des données issues des sciences basées sur l'expérimentation et l'observation. C'est ainsi qu'il a nourri sa pensée de ses propres recherches sur l'hypnose (Bergson, 1886) et sur les phénomènes de médiumnité (Evrard, 2016), tout en puisant dans les travaux psychologiques, neuropathologiques (en particulier ceux portant sur les aphasies), physiologiques et physiques de son temps (voir par exemple : Papanicolaou \& Gunter, 1987 ; Capek, 1971). Il avait une connaissance de première main des recherches sur le rêve et les états somnambuliques (Bergson, 1901), et il n'est donc pas surprenant qu'il ait intégré l'EMI au cours de la rédaction de Matière et mémoire, puisqu'une première étude systématique venait d'être entreprise par un autre philosophe français, Victor Egger (1896a, 1896b), en écho à d'autres cas précédemment publiés (Evrard et al., sous presse) et dans le prolongement de son étude sur la durée apparente des rêves (Egger, 1895).

\subsection{La mémoire panoramique}

Bergson cite les cas de suffocation brusque chez des rescapés de noyade et de pendaison. Dans la littérature psychologique française, Hyppolite Taine (1870) et Théodule Ribot (1881) en avait signalé quelques-uns, en s'appuyant principalement sur des observations réalisées à l'étranger. Mais l'intérêt pour ce sujet, manifesté par Egger au sein de la dynamique Revue philosophique de la France et de l'Etranger (Egger, 1885, 1886a) suscita la communication de nouveaux témoignages convergents. Deux aspects firent jonctions entre tous ces cas : la mémoire panoramique et ce qu'Egger baptisera le «moi vif».

La «rétrospective panoramique de la vie» (Haddock, 1851) ou "mémoire panoramique » (Noyes \& Kletti, 1977a) désigne une succession très rapide d'images rappelant des épisodes parfois oubliés de la vie personnelle du sujet, souvent avec hyperréalisme et luxe de détails. Ribot (1881, p.141) présente de tels exemples de « suractivité de la mémoire » dans le chapitre IV de son livre Les maladies de la mémoire, 
consacré aux "exaltations de la mémoire». Il privilégiait, comme beaucoup de ses collègues, une explication uniquement physiologique du phénomène. La vision des mourants ne ferait que traduire une multiplication ou accélération des états de conscience, amenant à revivre une succession de souvenirs sous la forme d'une simultanéité.

Toutefois, ces panoramas ne prennent pas uniquement la forme de successions rétrogrades marquant des étapes de la vie antérieure. Paul Sollier (1896) décrira en effet des panoramas similaires mais selon un déroulé prograde, anticipant sur les conséquences possibles du décès ou de la survie (baptisé ultérieurement "personal flashforwards » par le psychologue Kenneth Ring, 1984). Récemment, une étude sur ce phénomène chez des personnes ayant vécu ou non une EMI conclut qu'il nous apprend beaucoup sur les aspects temporels de la construction psychique (Katz, SaadonGrosman, \& Arzy, 2017). Elle suggère que la représentation des événements de la vie sous la forme d'un continuum serait présente chez tout-à-chacun, mais s'exprimerait de façon saillante dans des conditions extrêmes de stress psychologique et physiologique.

\subsection{L’accélération de la pensée}

Les sujets reconnaissent souvent l'aspect extraordinaire de cette revue détaillée de souvenirs effectuée à une vitesse folle et avec une forte intensité. Cela s'ajoute à d'autres aspects cognitifs, perceptuels et moteurs qui tranchent avec les modalités communes. Un point important est la distinction, déjà apportée à cette époque (Sollier, 1896), entre une expérience psychologique de l'agonie et la définition biologique de la mort. Si des récits d'EMI ont pu être apportés par des individus passés par un état de "mort clinique " (suspension temporaire de certaines fonctions vitales), aucun n'a pu être collecté suite à la mesure d'une "mort cérébrale" (observation d'une activité du cerveau égale à $0 \%$ ). Comme l'explique le neurologue Steven Laureys (2015, p. 147), spécialiste de cette question : "Un patient mort cérébralement ne revient plus à la vie. La mort cérébrale signifie tout simplement la mort. Mais la mort imminente ne signifie pas la mort. »

Dans son approche psychologique et philosophique de ces vécus, Egger supposait que l'idée d'une mort imminente était suffisante pour provoquer « un sentiment vif du moi » (Egger, 1896a, p. 28), phénomène qu'il comparait au rêve car, comme ce dernier, il est essentiellement constitué d'images. Toutefois, il affirmait que ce n'était pas un rêve, car son vécu, comme son souvenir, étaient marqués d'hyperlucidité et de véracité, ce qu'il chercha à signifier à travers le concept de moi vif (Le Maléfan, 1995). Cette "vivacité » désignait donc à la fois l'apparente vélocité de la pensée, son intensité et sa clarté. Une analyse d'un nombre important de cas d'EMI collectés par l'Université de Virginie permet de préciser les modalités de ce renforcement mental ressenti par une partie des sujets : $45 \%$ trouvent leur pensée plus claire que d'habitude, $37 \%$ la trouvent plus rapide, $29 \%$ la déclarent plus logique, et $19 \%$ affirment être mieux parvenus à la contrôler (Kelly et al., 2007, p. 386, note 16). Ces qualités furent particulièrement bien illustrées par la collection de cas constituée indépendamment par le géologue suisse Albert Heim (1892) où ces pensées riches, véloces et pragmatiques avaient vraisemblablement favorisé, occasionnellement, des gestes de sauvetage (Evrard, Toutain, Glazier, Le Maléfan, soumis). Généralement, cette accélération des pensées et cette succession intense de souvenirs dans l'espace psychique s'accompagnent d'un phénomène inverse: une perception ralentie du temps et des mouvements (Arstilla, 2012). 
Toutefois, revue panoramique de la vie et accélération de la pensée ne sont pas relatées par toutes les personnes affirmant faire ces expériences. L'EMI, devenue NDE avec Moody (1975), a effectivement connu une évolution de sa description phénoménologique, sans consensus sur les éléments qui la constituent. Actuellement, les chercheurs emploient l'échelle mise au point par le psychiatre Bruce Greyson (1983) qui évalue la présence ou l'absence pondérée de seize phénomènes contingents : distorsion temporelle; accélération des pensées; revue de vie antérieure; sentiment de tout comprendre; sentiment de paix ou de bien-être; sentiment de joie; sensation d'harmonie ou de communion avec l'univers; lumière brillante; intensité des sensations ; expériences extrasensorielles ; scènes du futur ; séparation du corps ; entrée dans un autre monde ; présence d'un être non-terrestre ; perception de défunts ; arrivée à une frontière ou un point de non-retour.

Un premier constat évident est que Bergson s'appuie uniquement sur une facette restreinte des EMI, principalement le vécu d'une pluralité de souvenirs dans une succession rapide suivant un sens rétrograde ou désordonné, et qui « signifie le moi qui va finir » (Egger, 1895a, p. 30) dont Egger faisait le cœur de son analyse. A nous d'examiner dans un premier temps la rencontre entre la théorie bergsonienne et cette forme étroite des EMI, avant de consacrer un second temps à examiner l'intégration possible d'autres aspects non traités par Bergson lui-même.

\section{La théorie des deux mémoires}

Dans son analyse de la perception (que nous ne développerons pas ici), Bergson montre que le passé paraît s'emmagasiner sous deux formes extrêmes : d'un côté, en tant que mécanismes moteurs qui utilisent ce passé pour agir; de l'autre, en tant qu'imagessouvenirs personnelles qui localisent tous les événements par leur contenu et leur place dans le temps. Il y a là une « différence profonde » (Bergson, 1896, p. 77) que, selon sa méthode de division des problèmes, Bergson érige au rang de différence de nature entre deux genres de souvenir. Il développe ensuite les propriétés de ces deux mémoires qui semblent aller en sens contraire, bien qu'elles nous apparaissent habituellement sous une forme impure combinant image-souvenir et mouvement.

\subsection{La mémoire pure}

L'analyse originale de Bergson procède de plusieurs postulats, qui lui semblaient étayés par les observations scientifiques de son temps, mais que l'époque contemporaine est loin de tenir pour acquis (Gallois \& Forzy, 1997). Le premier postulat est que le passé se conserve de lui-même, automatiquement et intégralement (Bergson, 1934, p. 189). Le deuxième est que tout souvenir spontané est tout de suite parfait (Bergson, 1896, p. 89) : le passé se conserve dans ses moindres détails sous la forme de souvenir-image. Le troisième postulat est qu'il n'y a pas d'oubli réel (Bergson, 1919, p. 76) mais uniquement des souvenirs impuissants. Ces trois postulats indiquent la présence d'une "mémoire pure » qui est une fiction scientifique capable de rendre compte d'un certain nombre de faits.

Bergson (1919, p. 76) tient la mémoire panoramique comme une illustration instructive de cette conservation totale du passé. D'autres illustrations proviennent des recherches sur le rêve, les états somnambuliques et l'hypnose, avec le rappel, dans ces états modifiés de conscience, de souvenirs exacts que le sujet ne se rappelait pas avoir enregistrés; ou encore l'exemple de la glossolalie par hypermnésie : «nous parlons des 
langues que nous ne nous souvenions même plus d'avoir apprises » (Bergson, 1896, p. 172).

La mémoire panoramique s'ajoute ainsi à d'autres exemples d'exaltations de la mémoire. Bergson en esquisse le mécanisme en identifiant, dans ces formes naturelles ou artificielles du sommeil, "un relâchement, au moins fonctionnel, de la tension du système nerveux, toujours prêt pendant la veille à prolonger l'excitation reçue en réaction appropriée » (Bergson, 1896, p.172). L'originalité de Bergson est donc de considérer que le facteur initial du phénomène d'hypermnésie est lié "non à une tension, à un raidissement, à une suractivité de l'âme, comme la plupart de ses prédécesseurs, mais, au contraire, à un relâchement de la tension habituelle de celle-ci » (Poulet, 1960, p. 31). Ce serait cette diminution ou déficience - cet événement tout négatif - et non l'accroissement ou l'apparition de quelque élément nouveau et positif, « qui provoque le jaillissement dans l'esprit de la chose la plus positive qui soit, le souvenir total, c'est-à-dire la saisie du moi par lui-même » (Poulet, 1960, p. 32). Cette mémoire désinhibée retrouve la force de franchir le seuil de la conscience et peut manifester le passé dans toute sa perfection, sans les habituels processus de filtrage qui en laissent une partie dans l'ombre. Bergson affiche clairement son dualisme, qu'il distingue des formes vulgaires de dualisme, en ce qu'il explique que cette mémoire immatérielle est autre chose qu'une fonction du cerveau et se rapproche le plus de ce qu'on pourrait entendre par « l'esprit » (Bergson, 1896, p. 266).

\subsection{La mémoire-habitude}

Cette première forme pure de mémoire est considérée par Bergson comme « la mémoire par excellence » (Bergson, 1896, p. 89), la « mémoire vraie» (Bergson, 1896, p. 167). Il désigne par "mémoire-habitude » l'autre facette du fonctionnement mnésique qui, si elle se coordonne toujours avec la première, n'en est pas moins strictement différenciée. La mémoire-habitude acquiert des souvenirs par répétition, comme dans l'apprentissage d'une leçon. Le rôle de la répétition est d'utiliser de plus en plus les attitudes corporelles associées à la formation des souvenirs, pour les organiser entre elles, et, « en montant un mécanisme, créer une habitude du corps » (Bergson, 1896, p. 89). La mémoire-habitude est constituée de l'ensemble de ces «mécanismes intelligemment montés qui assurent une réplique convenable aux diverses interpellations possibles » (Bergson, 1896, p. 167). Elle a donc un rôle adaptatif par rapport à la situation présente. Notre faculté de connaître correspondrait donc à « une puissance d'extraire ce qu'il y a de stabilité et de régularité dans le flux du réel »(Bergson, 1934, p.116) face à un environnement comportant une infinité de stimuli ${ }^{1}$.

A force de répétition, ces mécanismes - dits également systèmes ou schèmes sensorimoteurs - deviennent de plus en plus impersonnels, de plus en plus étrangers à notre vie passée (Bergson, 1896, p. 89). Le déploiement de la mémoire-habitude correspond davantage à celui d'un automatisme qui se soustrait à la représentation consciente: " Habitude plutôt que mémoire, elle joue notre expérience passée, mais n’en évoque pas l'image » (Bergson, 1896, p.167). D’où des réactions spontanées qui prolongent automatiquement, mais toujours plus ou moins adéquatement, des excitations venues du dehors, sans appui sur la conscience représentative.

\subsection{Le corps qui soude les deux mémoires}

\footnotetext{
${ }^{1}$ Pour une prolongation des intuitions de Bergson sur le fonctionnement de la mémoire-habitude, voir les lois de contingences sensori-motrices et les recherches récentes sur l'énaction (par exemple, Lenay, 2006).
} 
L'articulation de ces deux mémoires passe chez Bergson par une définition précise du rôle du corps, et plus particulièrement du cerveau :

« Le rôle du cerveau était de choisir à tout moment, parmi les souvenirs, ceux qui pouvaient éclairer l'action commencée, d'exclure les autres. Redevenaient conscients, alors, les souvenirs capables de s'insérer dans le cadre moteur sans cesse changeant, mais toujours préparé ; le reste demeurait dans l'inconscient. Le rôle du corps était ainsi de jouer la vie de l'esprit, d'en souligner les articulations motrices, comme fait le chef d'orchestre pour une partition musicale ; le cerveau n'avait pas pour fonction de penser, mais d'empêcher la pensée de se perdre dans le rêve ; c'était l'organe de l'attention à la vie. » (Bergson, 1934, pp. 89-90; ses italiques)

Ce passage évoque une "théorie du cerveau comme filtre » (Dunne, Jahn, Hoeger, Jones, 2009) dont on trouve des formulations voisines chez Myers, Schiller, William James, Huxley et des chercheurs contemporains (Kastrup, 2012 ; Smythies, 2011). Le cerveau est un outil de sélection au service d'un choix, en actualisant les souvenirs utiles et en maintenant dans le sous-sol de la conscience ceux qui ne serviraient à rien. De même pour la perception qui ne serait pas une formation mais une sélection d'images. La perception isolerait, dans l'ensemble de la réalité, ce qui nous intéresse : «elle nous montre moins les choses mêmes que le parti que nous en pouvons tirer " (Bergson, 1934, p. 168). Le corps serait « un lieu de passage des mouvements reçus et renvoyés, le trait d'union entre les choses qui agissent sur moi et les choses sur lesquelles j'agis » (Bergson, 1896, p.168; ses italiques). Ce passage introduit en particulier deux configurations dans lesquels l'articulation entre les deux mémoires prendra des formes différentes : celle de l'attention à la vie et celle opposée du désintéressement de la vie.

\subsection{L'attention à la vie}

Dans le cas le plus commun d'un individu tourné vers le présent, vers l'action, pris dans l'attention à la vie, Bergson décrit une cohésion entre les deux mémoires. Le corps va faire jouer les habitudes motrices qui sont dans son répertoire :

«Il peut reprendre des attitudes où le passé s'insérera; ou bien encore, par la répétition de certains phénomènes cérébraux qui ont prolongé d'anciennes perceptions, il fournira au souvenir un point d'attache avec l'actuel, un moyen de reconquérir sur la réalité présente une influence perdue (...) » (Bergson, 1896, p. 253-254)

Ce travail de la mémoire-habitude va être complété par celui de la mémoire vraie afin que l'être vivant puisse profiter de son expérience passée. Si l'habitude motrice va remonter vers les images semblables pour en extraire les similitudes, les images semblables vont redescendre vers l'habitude motrice pour se confondre dans un mouvement commencé ou un mot prononcé. La mémoire vraie aura pour «fonction première d'évoquer toutes les perceptions passées analogues à une perception présente, de nous rappeler ce qui a précédé et ce qui a suivi, de nous suggérer ainsi la décision la plus utile » (Bergson, 1896, p. 256). Dit autrement, cette mémoire se contracte « jusqu'à ne présenter que le tranchant de sa lame à l'expérience où elle pénétrera " (Bergson, 1896, p. 116). En plus de cette translation, par laquelle elle se porte tout entière audevant de l'expérience et se contracte ainsi plus ou moins, sans se diviser, la mémoire intégrale fait un mouvement de rotation sur elle-même, " par lequel elle s'oriente vers la situation du moment pour lui présenter la face la plus utile » (Bergson, 1896, p. 192). 
Il y a donc une double attraction qui part à la fois des habitudes du corps et de l'activité de l'esprit. D'un côté, l'état sensori-moteur oriente la mémoire vraie ; et d'autre part, cette mémoire elle-même, avec la totalité de notre passé, exerce une poussée en avant pour insérer dans l'action présente la plus grande partie possible d'elle-même (Bergson, 1896, p. 192). Pour en donner une sorte d'illustration à grande échelle, si, après être entré dans une pièce, on se rend compte que l'on a oublié ce qu'on venait y faire, franchir le seuil à rebours nous place dans l'attitude corporelle qui nous remet en contact avec le souvenir égaré (Radvansky, Krawietz \& Tamplin, 2011). L'action résulte d'une coordination entre ce qu'appelle l'état sensori-moteur et ce que propose la mémoire intégrale.

Nous pouvons figurer le processus de l'attention à la vie sous la forme de trois étapes [voir Figure 1] :

1) La survenue d'une perception emmagasinée sous une forme passée (souvenirs personnels) et une forme présente (habitudes motrices) ;

2) Une contraction des deux mémoires : la mémoire vraie fait un mouvement de rotation et de translation; la mémoire habitude opère une sélection en fonction de l'état sensori-moteur.

3) La zone contractée réunit les souvenirs utiles, à la fois sous forme de représentations et de schèmes, et inhibe les souvenirs impuissants. Il en naît un ordre d'action. 
Figure 1: Trois étapes de l'attention à la vie

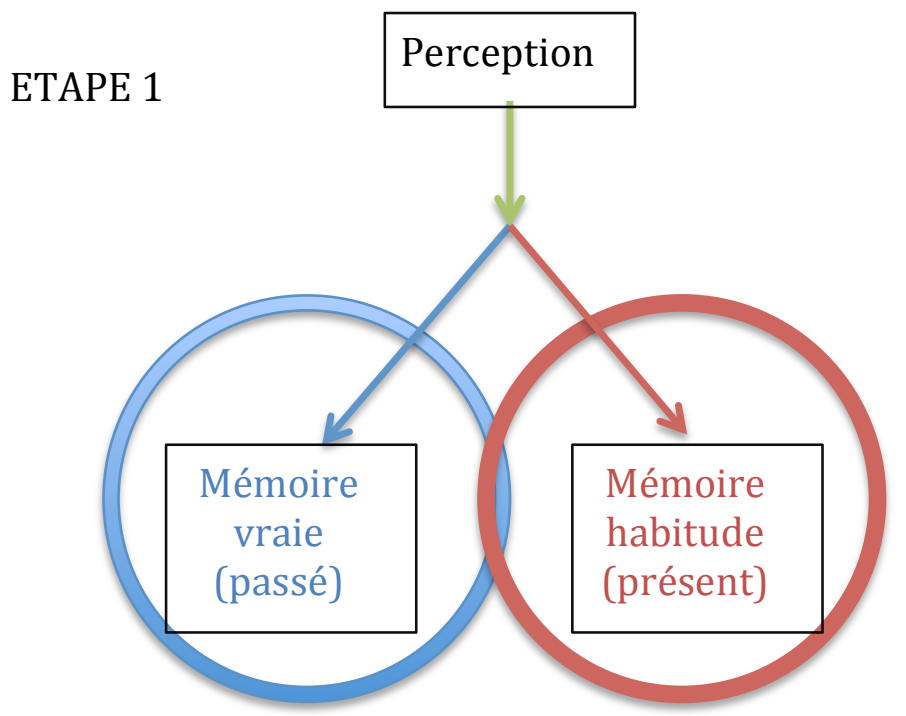

ETAPE 2

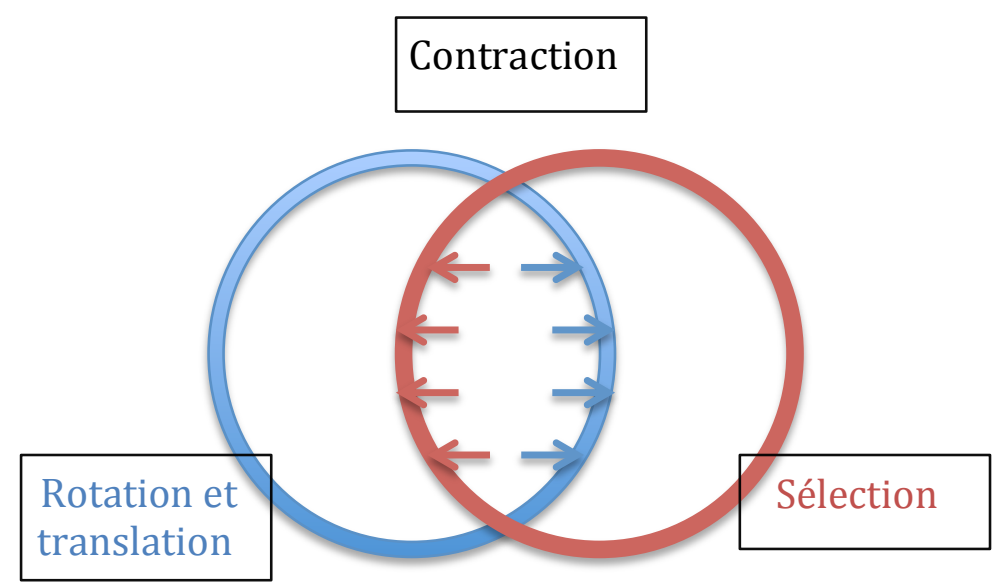

ETAPE 3

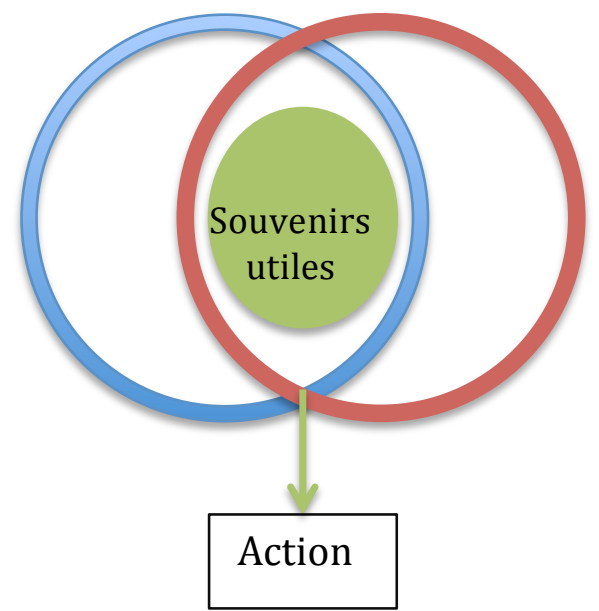


La distinction de ces deux mouvements clarifie les situations où l'attention et la mémoire sont troublés. Bergson explique astucieusement les perturbations de certaines fonctions de l'attention et de la mémoire par des lésions de la reconnaissance qui, en aucun cas, n'impliquent la destruction des souvenirs (Bergson, 1896, p. 118). Ces lésions tiennent à deux causes :

« tantôt à ce que notre corps ne peut plus prendre automatiquement, en présence de l'excitation venue du dehors, l'attitude précise par l'intermédiaire de laquelle s'opérerait une sélection entre nos souvenirs, tantôt à ce que les souvenirs ne trouvent plus dans le corps un point d'application, un moyen de se prolonger en action. » (Bergson, 1896, p. 118).

Toutefois, Bergson distingue une autre configuration où l'attention à la vie n'est plus première, et dont l'archétype est le rêve.

\subsection{Le désintéressement de la vie}

L'attention à la vie n'est pas une constante : c'est plutôt une variable dont le revers est le désintéressement de la vie. Le cerveau viendrait canaliser notre attention dans la direction de l'avenir, pour la détourner du passé (Bergson, 1934, p. 189), mais la tendance inverse nous ramène vers cette partie de l'histoire qui n'intéresse pas notre action présente. La mémoire est dans une situation de tension qui l'incline à s'insérer dans l'action présente ou à s'en détacher. Bergson parle alors de degrés ou de tons différents de la vie mentale (Bergson, 1896, p. 7). Lors de la détente de cette tension, la mémoire régresse, se dilate et se désolidarise de la coordination avec le corps. Sa finalité n'est plus l'action mais la contemplation : elle circule sur le «plan du rêve $»^{2}$.

Nous pouvons figurer le processus du désintéressement de la vie sous la forme de trois étapes [voir Figure 2] :

1) La survenue d'un déséquilibre sensori-moteur, qui peut être d'origine psychologique ;

2) Une dilatation des deux mémoires : la mémoire vraie se détache du présent ; la mémoire habitude relâche son exigence pratique.

3) Il en émerge une contemplation où une représentation du passé prend le pas sur le présent.

\footnotetext{
${ }^{2}$ Dans la figuration bergsonienne d'un psychisme sous forme de cône, le «plan du rêve » est le plan de conscience « le plus étendu de tous, sur lequel est étalé, comme sur la base d'une pyramide, tout le passé de la personne, jusqu'au point, comparable au sommet, où la mémoire n'est plus que la perception de l'actuel avec des actions naissantes qui la prolongent » (Bergson, 1934, p. 81). Il entre en intersection avec le «plan de l'action » à l'endroit du corps. En dépit de cette évocation du « rêve », qui en est une illustration, ce plan de conscience ne se réduit pas à l'activité onirique.
} 
Figure 2 : Trois étapes du désintéressement de la vie ETAPE 1

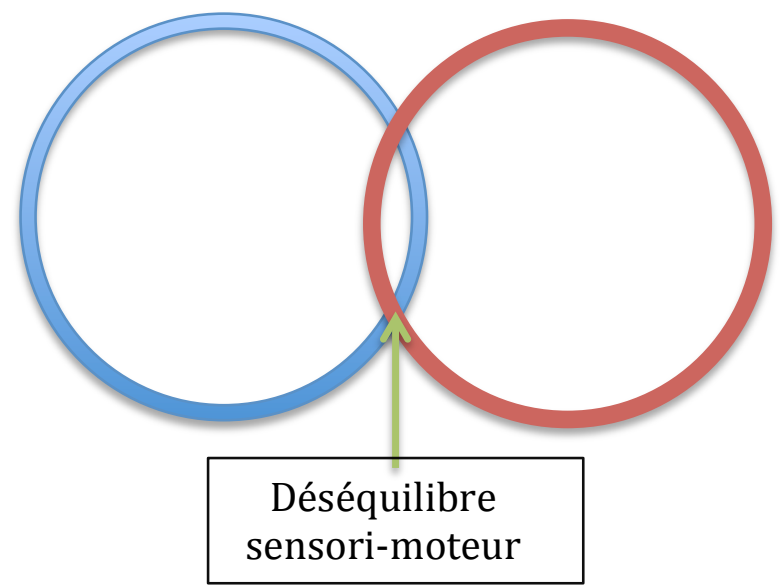

ETAPE 2

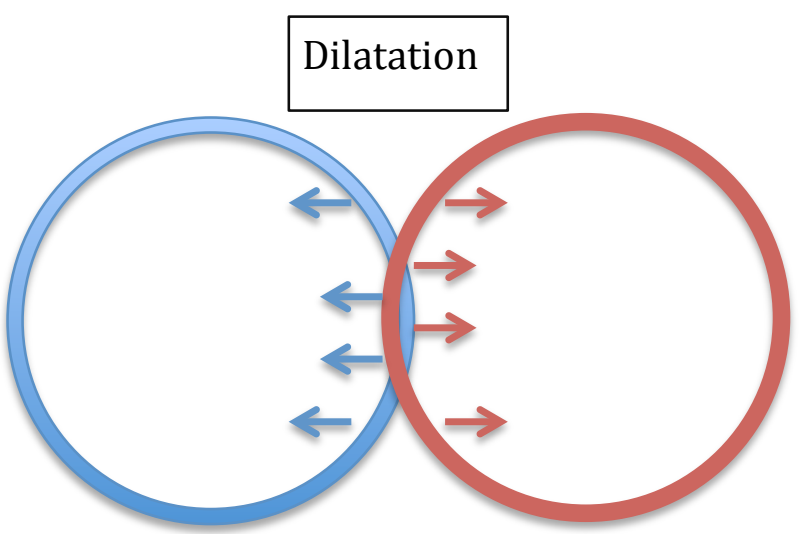

ETAPE 3

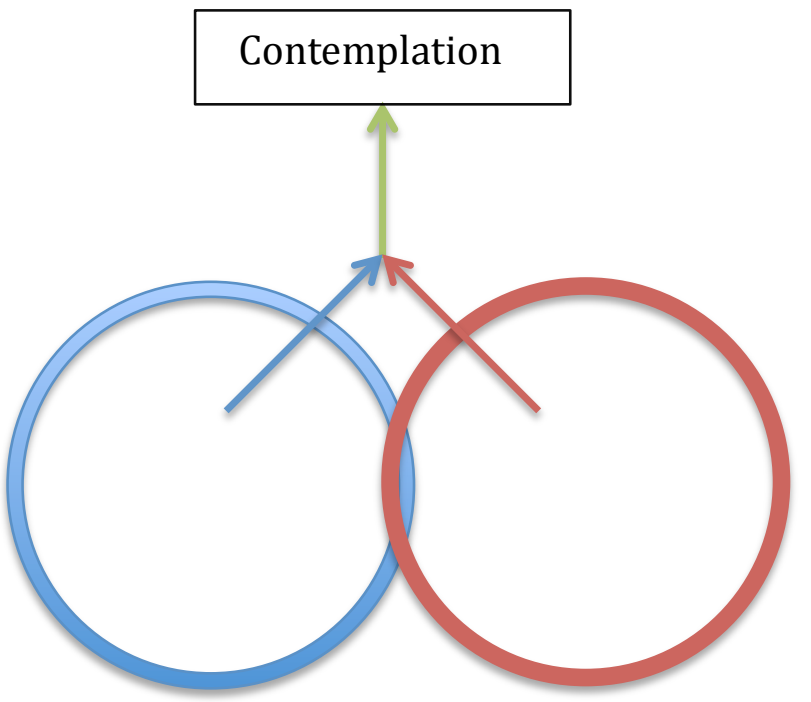


Pour pouvoir s'abstraire de l'action présente, il faut savoir attacher du prix à l'inutile. Bergson pense qu'il se pourrait bien que seul l'homme soit capable d'un tel effort (Bergson, 1896, p. 87-88). Il donnera des exemples vertueux d'hommes "moins adhérents à la vie » : les mystiques (Bergson, 1932), les artistes (Bergson, 1934, p. 168), les adeptes de la métaphysique ou de l'intuition (Bergson, 1934, p.170). Mais son exemple le plus trivial est le rêveur: le temps du sommeil est un moment d'extrême « inattention à la vie ». Ce relâchement psychique permet à la masse innombrable des sensations éprouvées, associées directement aux souvenirs passés accumulés, de revenir sous une forme à la fois déguisée et proliférante. Le "mystère » du rêve tient donc au fait que le "moi du rêveur » est stupéfait à son réveil par l'actualisation de souvenirs sensibles complètement et rapidement oubliés par la conscience diurne. Comme le dit Bergson (1901) dans sa conférence à l'Institut général psychologique, le rêve contient «toute la vie mentale » - dont l'intégralité de ses souvenirs passés - «à l'exception de l'attention à la vie». De là, l'instabilité et l'absurdité mais aussi la vivacité et la production inouïe de l'activité onirique.

En plus de cette analyse du rêve, Bergson a recours aux observations d'exaltations de la mémoire comme autant de preuves en faveur de sa théorie. Examinons à présent ces points de convergence.

\section{Les exaltations de la mémoire}

Bergson énumère différentes manifestations des souvenirs inconscients (fausse reconnaissance, rêve, somnambulisme) qui le conduisent à une saisie plus approfondie du fonctionnement du psychisme. Le point commun de ces phénomènes est l'exaltation du souvenir : la conscience se dilate en un relâchement qui supprime l'activité sélective à l'œuvre dans la perception. Il y a alors profusion de sens.

\subsection{Le déséquilibre sensori-moteur qui génère l'exaltation}

C'est comme illustration supplémentaire de cette exaltation que le philosophe, dans Matière et mémoire, fait appel aux observations, résumées par Ribot (1881) et Egger (1896a, 1896b), de vécus étranges de rescapés de noyade et de pendaison : " Le sujet, revenu à la vie, déclare avoir vu défiler devant lui, en peu de temps, tous les événements oubliés de son histoire, avec leurs plus infimes circonstances et dans l'ordre même où ils s'étaient produits » (Bergson, 1896, p. 172). En 1913, il manifeste une connaissance plus complète du phénomène ${ }^{3}$ puisqu'il le dissocie du symptôme d'asphyxie : «il se produira aussi bien chez un alpiniste qui glisse au fond d'un précipice, chez un soldat sur qui l'ennemi va tirer et qui se sent perdu » (Bergson, 1919, p. 77). L'origine psychologique du déclenchement ne fait dès lors plus aucun doute pour lui et il se propose d'en éclairer le mécanisme.

De tels faits viennent, selon lui, confirmer son hypothèse de deux mémoires indépendantes, formulée ainsi : «nous devrons constater une exaltation de la mémoire spontanée dans la plupart des cas où l'équilibre sensori-moteur du système nerveux sera troublé, une inhibition au contraire, dans l'état normal, de tous les souvenirs spontanés qui ne peuvent consolider utilement l'équilibre présent » (Bergson, 1896, p. 91). Dans cette hypothèse, la notion d'équilibre sensori-moteur n'est pas clairement

\footnotetext{
3 Nous nous inscrivons en faux contre l'affirmation de Poulet (1960, p. 23) selon laquelle cette conférence ne montre aucune évolution dans l'analyse par Bergson du thème de la vision panoramique de la vie.
} 
précisée et laisse place à une certaine ambiguïté, puisqu'elle s'appliquerait également à des cas où le déséquilibre est généré uniquement par un changement psychologique.

En effet, en 1913, Bergson attribue la vision panoramique du passé à un "brusque désintéressement de la vie né de la conviction soudaine qu'on va mourir à l'instant " (Bergson, 1919, p. 77). Contre la tendance naturelle de la vie à se porter vers l'avant, contre le mécanisme cérébral qui a précisément pour fonction de nous masquer le passé en obscurcissant tous nos souvenirs sauf un - le souvenir utile -, il y aurait des occasions où l'esprit " se détend et par là même se retourne en arrière ; il y retrouve son histoire " (Bergson, 1919, p. 77). Cette occasion n'est pas un simple affaiblissement de l'attention à la vie, mais une véritable « conversion brusque de l'attention » (Bergson, 1934, p. 170). Poulet (1960) y voit un retournement de l'acte d'attention d'un pôle positif à un pôle négatif, dans un mouvement rétrograde de la pensée. Le mourant deviendrait le « rêveur absolu » qui, "désintéressé de toute action, soustrait à l'inhibition que constituaient pour lui les nécessités de la vie présente, [reprendrait] sans effort possession de son passé et de son moi » (Poulet, 1960, p. 33).

\subsection{L'attention de l'espèce}

Toutefois, plusieurs aspects nous font douter de la possible inscription de ce phénomène dans la simple continuité de la distraction, de la rêverie et du rêve comme étapes successives d'un long glissement de l'esprit. D'une part, tout intérêt pour l'action n'est pas perdu, comme s'en rend compte Poulet (1960, p. 31) puisqu'il associe le vécu de cette détente à un moment précis de l'expérience, lorsque le sujet a l'impression de capituler. Nous reviendrons sur cet aspect lorsque nous explorerons la "conscience hyperincorporée». D’autre part, dans sa conférence de 1913, Bergson distingue clairement l'attention ici impliquée de l'attention volontaire, qui est momentanée et individuelle. Il repère ce qu'il appelle alors «l'attention de l'espèce », présentée comme une "attention constante, commune à tous, imposée par la nature » (Bergson, 1919, p. 77) ${ }^{4}$. Ce concept a été forgé plusieurs années auparavant puisqu'on le trouve dans l'article «Le souvenir du présent et la fausse reconnaissance » écrit en 1908 (Bergson, 1919), où il désigne une sorte de socle commun à une espèce sur lequel va se superposer l'attention générale (c'est-à-dire volontaire). Mais il n'y a pas d'autre occurrence de cette expression dans toute l'œuvre de Bergson.

Si le philosophe convoque un concept de registre supérieur, c'est qu'il ne s'agit donc pas d'un relâchement identique à celui du sommeil, car, si ce processus va dans la même direction, il le fait différemment. On pourrait mettre en lien cette notion plus fondamentale et spécifique d'«attention de l'espèce» avec sa notion d'élan vital (Bergson, 1907) laquelle est en contradiction avec la conviction de la mort acquise par l'homme suite à ses réflexions intellectuelles. Selon le philosophe, la nature va réagir à cette pensée de la mort inévitable en lui opposant l'image d'une continuation de la vie après la mort qui remet les choses en ordre dans le champ de l'intelligence. Dans une

\footnotetext{
$4 \quad$ Puisque plusieurs versions du même texte ont circulé, il faut noter que le texte de la conférence de 1913 (donnée en français) qui a été publié en premier dans les Annales des sciences psychiques, puis traduit en anglais par H. Wildon Carr dans les Proceedings de la SPR en 1914-1915, présente des différences avec la version de 1919. L'attention de l'espèce (racial attention en anglais) est comparée à « l'attention volontaire (...) qui dépend de l'individu » (Bergson, 1913, p. 327) et est décrite simplement comme "l'attention qui s'impose à l'homme normal ». Le texte de 1919 ajoute donc sa constance et sa source naturelle. De même, la « conviction soudaine qu'on va mourir à l'instant » remplace une formule plus hésitante où le désintéressement de la vie est "produit dans certains cas par la menace d'une mort subite» (Bergson, 1913, p. 327). Bergson se fait donc plus subjectiviste et plus affirmatif en 1919. Je remercie Pierre Lagrange pour ses précisions historiques.
} 
lecture sociologique, on aboutit ainsi à l'origine de ce que Bergson appelle la religion statique, une religion d'essence sociale qui n'est susceptible d'aucune créativité dans l'ordre moral, et qu'il oppose à la religion dynamique, ou mysticisme, d'essence plus individuelle (Bergson, 1932). Il ne manque d'ailleurs pas d'auteurs pour lier les imaginaires déployés dans les EMI avec les grandes traditions religieuses (Coppes, 2013). Dans une lecture psychologique, ce sursaut d'élan vital pourrait également correspondre à cette réaction instinctive qui fait obstacle à la conviction ponctuelle de la mort inévitable, de l'anéantissement (Roisin, 2010).

\subsection{Une expérience de la mémoire pure}

Une autre convergence semble apparaître entre la théorie bergsonienne et la mémoire panoramique, même si elle n'est pas soulignée explicitement par Bergson. Elle concerne la nature des souvenirs qui émergent à cette occasion. Puisque la mémoire se divise à chaque instant en deux directions, l'une orientée et dilatée vers le passé, l'autre contractée en vue de l'avenir, les souvenirs s'échelonnent de façon graduée entre ces deux pôles. Il y a, chez Bergson, une multiplicité des niveaux possibles de mémoire. Chacun de ces niveaux comprend non pas tel ou tel moment du passé mais sa totalité, puisque la mémoire est un tout indivisé. Mais, comme le dit Deleuze (1966, p. 56), « il comprend cette totalité à un niveau plus ou moins dilaté, plus ou moins contracté ». Bergson place les souvenirs personnels, dont la série dessine le cours de notre existence passée, dans la dernière et plus large enveloppe de notre mémoire (Bergson, 1896, p.116). Les enveloppes suivantes sont plus étroites, mais supportent les mêmes souvenirs sous une forme diminuée, « de plus en plus éloignés de leur forme personnelle et originale, de plus en plus capables, dans leur banalité, de s'appliquer sur la perception présente et de la déterminer à la manière d'une espèce englobant l'individu » (Bergson, 1896, p. 116). Il s'agit donc, avant la lettre, d'une vision hologrammatique du souvenir, chaque souvenir correspondant à une information redondante sur la totalité de la mémoire, mais qui apparaît dans une définition d'autant moins nette qu'il est extrait d'une enveloppe de taille étroite. Bergson représente ainsi la mémoire vraie par un cône dont l'infinité de segments sont des cercles concentriques qui se resserrent jusqu'à former un point - notre corps - dans leur intersection avec le plan de l'action.

L'intérêt de cette description de Bergson est qu'elle s'ajuste avec le phénomène de mémoire panoramique, dans lequel le sujet semble atteindre la couche la plus personnelle de son être. D'une façon ordonnée, il contemple mais aussi revit les grandes étapes constituantes de son identité propre. Cette exaltation le mène-t-il effectivement jusqu'à l'enveloppe la plus large de sa mémoire ? L'autre versant du phénomène, à savoir le panorama prograde anticipant la vie à vivre ou les conséquences du décès, viendrait suggérer que la perception de notre destinée, des étapes futures où nos choix nous conduisent, fait également partie du cœur de notre personnalité. Cela n'entre pas en contradiction avec la théorie bergsonienne qui fait de la mémoire la « synthèse du passé et du présent en vue de l'avenir » (Bergson, 1896, p. 248).

Sans le dire directement, Bergson fait coïncider la mémoire panoramique avec l'expérience de la mémoire pure. Il s'agirait d'un de ces cas exceptionnels, prévus par sa théorie, où s'isole et se manifeste pleinement « une mémoire toute contemplative qui n'appréhende que le singulier dans sa vision» (Bergson, 1896, p. 173 ; ses italiques). Cette mémoire rendue puissante serait celle de l'être humain qui rêverait son existence au lieu de la vivre et qui tiendrait « sous son regard, à tout moment, la multitude infinie des détails de son histoire passée » (Bergson, 1896, p. 173). 


\section{Conclusion intermédiaire}

Bergson a développé sa théorie des relations entre l'esprit et la matière à partir d'une analyse fine de données scientifiques de son époque. Pour expliquer le fonctionnement normal, il s'est inspiré de la psychologie pathologique en allant chercher, par exemple dans les aphasies, des cas dans lesquels il pouvait isoler des processus habituellement interpénétrés. Pour faire tenir sa vision d'une mémoire à la fois extrêmement contractée dans l'action normale, et extrêmement dilatée dans sa forme pure, il a puisé dans certaines anomalies du fonctionnement mnésique. Ces anomalies ne relevaient plus de l'infra-normal, à la manière des pathologies de la mémoire et de l'attention, mais du supra-normal, c'est-à-dire des formes exaltées de la mémoire qui sont soit provoquées artificiellement par l'hypnose ou des drogues, soit spontanées comme dans l'EMI.

Dans Matière et mémoire, la vision panoramique de la vie est ramenée au rang d'une illustration supplémentaire de son modèle. Mais la conférence Fantômes de vivants est l'occasion de souligner la nature particulière du déclencheur de ce vécu, qui ne correspond ni à la physiologie de l'asphyxie, ni au relâchement fonctionnel similaire au sommeil. Il s'agit d'une rupture plus fondamentale qu'un simple dérangement dans l'équilibre maintenu par le cerveau entre l'excitation extérieure et la réaction motrice. La conviction soudaine de la mort propre éveille «l'attention de l'espèce » qui lui fait barrage. On perçoit ainsi que l'EMI finit par mettre au travail la pensée de Bergson, même si celui-ci ne livrera pas explicitement le fruit de ses conclusions dans la suite de ses travaux.

Si l'on souhaitait se restreindre à une analyse historique, il serait légitime de s'arrêter à l'endroit où Bergson semble abandonner la question. Toutefois, nous souhaitons explorer plus avant les points de convergence et de divergence entre la théorie bergsonienne et les EMI, en nous appuyant sur d'autres éléments théoriques que Bergson n'a pas mobilisés dans son analyse succincte; et sur d'autres données à propos des EMI dont Bergson n'avait pas pris connaissance. L'ensemble pourrait nous convaincre que la théorie de Bergson est confirmée empiriquement par les EMI au-delà de ce qu'il envisageait en son temps, ce qui par conséquent nous amène à considérer l'actualité du modèle bergsonien dans la compréhension des EMI.

\section{Prolongements de l'approche bergsonienne des expériences de mort imminente}

\subsection{L'expérience de la mémoire pure ou la conscience désincorporée}

La méthode de Bergson, combinant données scientifiques et intuition philosophique, pourrait décontenancer. Mais il est également possible de saisir les objets de son intuition comme des vérités empiriques en attente de confirmation. C'est ce qu'il semble faire lorsqu'il assimile le tout jeune phénomène de la mémoire panoramique à un contre-exemple parfait du fonctionnement habituel de la mémoire, c'est-à-dire comme un trouble de l'encorporation. Par ses fictions philosophiques de la mémoire pure et de la perception pure, Bergson ouvre un espace virtuel, comme une expérience de pensée, où il dépose ses hypothèses sur les phénomènes qui devraient y être observables. Que nous dit-il alors de l'expérience de la mémoire pure? Quelles correspondances peut-on établir avec le riche vécu de l'EMI?

Dans le segment correspondant à la mémoire pure, le point essentiel est que l'individu se détache de son état sensoriel et moteur (Bergson, 1896, p.181). Cette propriété 
caractéristique nous fait l'envisager comme une conscience désincorporée, à la manière des spécialistes de la dissociation somatoforme (Irwin, 2000), mais sans l'extraire totalement de sa dépendance au corps vivant et à sa localisation (contrairement à : Fracasso \& Friedman, 2011). Cette hypothétique conscience désincorporée ne serait pas tant un état que l'horizon d'un processus de distanciation entre le sujet et l'image de son corps (Le Maléfan \& Coq, 2015). Des marques de cette distanciation vers une conscience désincorporée sont repérables dans une grande partie de la phénoménologie des EMI, au niveau sensoriel, émotionnel, de l'incorporation, de la mémoire, de la pensée, de l'identité et de la perception du temps.

- Sensations : Au niveau sensoriel, ce constat est à la fois courant et ancien : Sollier (1896) et Egger (1896b) observaient une suppression de la sensibilité générale, l'EMI se traduisant sur le plan somatique par une anesthésie et une analgésie, déjà décrite par Heim (1892). Le sujet ne retrouvait ses sensations qu'au retour de son expérience ${ }^{5}$. Dans une étude prospective sur des EMI survenant lors d'arrêts cardiaques, $10 \%$ des sujets relataient encore une sensation plus ou moins forte de douleur (Schwaninger et al., 2002), ce qui montre que cette analgésie n'est pas toujours complète.

- Emotions : Bergson (1896, p. 193) identifiait le corps à «ce qui fixe notre esprit, ce qui lui donne le lest et l'équilibre». Délesté de ce corps, quels seraient nos éprouvés? Lors des EMI, les sujets rapportent des ressentis émotionnels paradoxaux, à la fois intenses et neutres, proprement ineffables selon le vocabulaire usuel. Les chercheurs recensent ainsi des sentiments de joie, d'harmonie, de compréhension universelle, d'esthétique, de grâce, de perfection, que le sujet ne parvient pas à comparer avec son répertoire de «sensations terrestres » (Jourdan, 2007). Cela serait-il dû au fait que ce souvenir pur est délié de sa contrepartie incarnée?

- Incorporation : L'impression de désincorporation est tout à fait envisageable dans le cadre de cette distanciation. Cette portion "autoscopique » de l'EMI donnée pour équivalent à l'expérience de hors corps (EHC) - est souvent présentée comme le premier temps de l'expérience (Sabom, 1982 ; Jourdan, 2007), bien qu'elle ne soit pas systématique (seulement $24 \%$ des sujets dans l'étude prospective de van Lommel et al., 2001). Une étude récente sur 154 récits d'EMI en français a évalué la chronologie des éléments de l'EMI de façon empirique et a montré que l'EHC est la dimension qui est la plus fréquemment placée au début des récits (mais uniquement dans $35 \%$ d'entre eux) (Martial et al., 2017). Le sujet passe d'une perspective où ses représentations du monde extérieur sont nourries par le circuit classique de la perception à une perspective où le rôle du corps paraît nul. Ce dernier, lorsqu'il est perçu, est réduit à n'être qu'une image parmi les autres, image-objet dont le sujet ne saisit pas toujours d'emblée la familiarité. Alors que la perception opérait une sélection drastique des images sur lesquelles agir, ce procédé apparaît ici caduque (Jourdan, 2007) : les sujets disent tout percevoir avec une précision infinie ; ils peuvent contempler une fleur comme s'il s'agissait de l'expérience esthétique la plus importante et originale de leur vie. Ils affirment passer d'une perception à une autre par une succession ne répondant pas à des contraintes matérielles, mais uniquement aux fluctuations de leur volonté, comme dans un rêve. Leur champ de vision, qui n'est

\footnotetext{
5 Une étude récente a montré que la perception de la douleur diminuait durant l'induction expérimentale d'une illusion de sortie hors du corps (Hansel et al., 2011).
} 
plus restreint par un angle oculaire, recueille des données en provenance de toutes les directions. Leur regard pénètre la matière et la traverse même. Ils circulent entre les images en allant de l'infiniment petit à l'infiniment grand. Le fait même de parler du sens de la vue est une erreur puisque le ressenti est multimodal, voire transmodal. Ring et Cooper (1999) parlent de « mindsight » à partir des récits sensoriels d'EMI effectués par des aveugles de naissance. De plus, le passage d'une perception contractée à une vision dilatée pourrait correspondre à la traversée d'un couloir ou d'un tunnel qui est quelquefois rapportée.

- Mémoire : Les recherches récentes examinent la qualité des souvenirs d'EMI et semblent confirmer leur association à une impression d'hyperréalisme (Moore \& Greyson, 2017). Cela est paradoxal car le sujet se souvient, pour l'un, s'être promené dans un jardin paradisiaque, pour l'autre, avoir traversé les flammes de l'Enfer... Ce réalisme est utilisé par certains comme un indice de la validité ontologique de ces visions (Moody, 1975). Or, dans la théorie bergsonienne, il reste compatible avec le statut de vision illusoire, puisqu'il est prévu que les souvenirs de niveaux supérieurs, ceux qui se rapprochent le plus de la mémoire pure, soient chargés de qualités, de contenus, de couleurs, qui en restituent la « haute définition ». Chez Bergson (1896, p. 265), le souvenir est tout le contraire d'une perception affaiblie.

- Pensée : A proximité de la mémoire pure, la pensée est de plus en plus régie par l'association libre des idées. Elle n'est plus sélectionnée en vue d'une action concrète. L'utilité vitale qui contraint les associations par ressemblance et par contiguïté opère moindrement. Les connexions sémantiques prédominent dans l'organisation du réseau des images. Ce degré supplémentaire de liberté pourrait correspondre au « moi vif » décrit par Egger, c'est-à-dire à la vélocité, la clarté et l'intensité des pensées. A la fluidité des images s'ajoute l'apparente lucidité du raisonnement.

- Identité : Poulet (1960, p. 35) a produit une fine analyse de l'évolution des considérations de Bergson au sujet de la vision panoramique. Si Bergson a d'abord hésité à penser, comme ses contemporains, que le panorama de vie pouvait se décrire comme une multiplicité quantitative de souvenirs distincts, juxtaposés, il a ensuite avancé qu'il correspondait davantage à une multiplicité qualitative de données qui s'entre-pénètrent, se fondent mutuellement l'une dans l'autre. Les images qui défilent ainsi sont «multiplicité sans divisibilité et succession sans séparation » (Bergson, 1922, p. 55), une "continuité mélodique de l'existence» (Bergson, 1889), une «phrase unique semée de virgules, mais nulle part coupée par des points » (Bergson, 1919, p. 57) qui ne manque pas de faire énigme pour les sujets. Poulet affirme que cette vision panoramique serait une forme très basse d'expérience de la durée, puisqu'elle "aboutit à l'éparpillement de ce qu'elle restitue » (Poulet, 1960, p. 37-38). Le passé ainsi étalé serait « du temps spatialisé, figé, du temps presque matérialisé » (Poulet, 1960, p. 37-38), soit l'inverse même de ce que Bergson entend par durée. Poulet ne prend pas en compte cet élément, fréquemment rencontré dans les récits d'EMI, des utilisations positives de cette revue de vie pour jauger voire refondre l'identité. Loin d'être présentée " en mille morceaux », l'identité est alors abordée dans une perspective transversale. Il s'agit moins d'un "temps transformé en espace» (Poulet, 1960, p. 37) que d'une dimension d'espace qui permet de naviguer dans les trois dimensions du temps (passé, présent et futur). 
- Perception du temps : Dans de nombreux récits d'EMI, le temps est perçu de manière déformée. Le monde extérieur semble tourner au ralenti, tandis que la vie intérieure est fulgurance. Cela pourrait correspondre à un accroissement de durée lié à la distanciation, l'esprit étant alors moins soumis au rythme du corps, à la nécessité d'entrer dans le présent de l'action. La distanciation correspond également à un « détachement du présent » (Bergson, 1896, p. 81). L'épaisseur de durée allant croissante, la mémoire se dilatant, la conscience désincorporée élargit son empan pour embrasser en un seul regard plus de pensées et de souvenirs qu'elle ne le fait communément, lorsqu'elle se contracte en vue de l'action.

En somme, l'expérience de la mémoire pure est comparable à celle du rêve, sans s'y réduire, comme le suggérait déjà Egger (1895a). Avec Bergson, nous pourrions dire que l'EMI explore le plan du rêve, mais en ayant perdu certains des liens qui, en dépit de tout, maintiennent le rêveur en contact avec son corps et son environnement présent. Cette déliaison plus radicale pourrait s'expliquer par la disjonction fondamentale évoquée par Bergson, mais dont il n'a pas établi toutes les conséquences.

Quand le sujet change de cap, lorsqu'il se tourne non plus vers le plan de l'action mais vers le plan du rêve, il se distancie du point d'intersection de ces plans que constituait son corps. Ce faisant, il bénéficie progressivement de toutes les propriétés de la mémoire pure en se défaisant petit à petit des contraintes pratiques habituelles. Cette libération découpe un réel que certains assimilent à une réalité spirituelle plus authentique, mais qui n'en reste pas moins un écart avec le règne du vivant. La notion de conscience désincorporée tente de rassembler une grande partie de la phénoménologie de l'EMI à travers une lecture bergsonienne. Elle n'implique pas nécessairement une discussion des prolongements de cette conscience désincorporée, dans une survie indépendante du corps vivant, discussion néanmoins abordée par Bergson dans sa conférence de 1913 et d'autres textes (Bergson, 1919).

\subsection{L’expérience de la perception pure ou la conscience hyperincorporée}

Certaines recherches dans le champ des EMI ont mis en évidence des anomalies dans le registre de la perception et de la motricité, qui sont toutefois moins bien répertoriées que les autres anomalies (Evrard et al., sous presse ; Evrard et al., soumis). Le psychiatre Russell Noyes et ses collègues ont ainsi interrogé de nombreuses personnes ayant eu un vécu étrange lors d'une confrontation avec un danger potentiel. Ils ont repéré une forme de «dépersonnalisation» par laquelle les sujets se «coupaient» de leurs ressentis corporels pour ne plus éprouver ni la douleur ni la peur de la mort (Noyes \& Kletti, 1976a, 1976b, 1977a, 1977b). Ce processus s'accompagnait, chez $60 \%$ des sujets, de l'impression que leurs mouvements et leurs pensées fonctionnaient de manière automatique, et près de $40 \%$ d'entre eux ont même affirmé qu'ils s'étaient sentis sous le contrôle d'une force externe. Ce ressenti d'un mode "autopilote » était généralement accompagné d'un sens d'invulnérabilité réconfortant et de l'impression d'un contrôle magique sur les choses. Noyes et Kletti (1976b, p.106) concluaient que cette « dépersonnalisation » semblait être une réaction universelle face à un danger menaçant la vie et un pattern adaptatif de base adopté par le système nerveux, similaire aux réactions « se battre ou fuir » mises en évidence par Cannon (1915).

Ces automatismes sont rarement poussés au premier-plan dans la littérature sur les EMI, et l'une des raisons pourrait être leur manque de saillance dans les témoignages. En effet, le sujet perd souvent la conscience et la maîtrise des actions entreprises avec son 
corps. Plusieurs des mouvements réalisés en état d'automatisme sont en fait relatés par des témoins de la scène ou déduits dans l'après-coup par le sujet, lorsqu'ils constatent ses blessures ou sa position décalée dans l'espace (Evrard et al., sous presse). Une autre raison est que de nombreuses EMI sont associées à un état de passivité physique, par exemple sous anesthésie sur un lit d'hôpital, sans que ce soit une règle absolue. De nombreux cas combinent EMI et activité physique, le corps continuant de se mouvoir, de façon cohérente et adaptée, reproduisant parfois à la perfection les schèmes sensorimoteurs auparavant appris. Alvarado (2000) cite le cas d'une policière débutante qui, sortie de son corps après un moment de panique, observa depuis un lieu surélevé son propre corps en train de maîtriser un suspect dans le pur respect de sa formation. Cette coordination exceptionnelle de l'esprit et du corps est également décrite dans les " expériences de flux » (Csíkszentmihályi, 1990) et chez les sportifs (Murphy \& White, 1995), en particulier dans les sports extrêmes où sont observés des ajustements extrêmement fins et rapides pour contrôler conjointement le corps et l'équipement, en réaction à des perceptions vives et détaillées de la position du corps et de l'espace environnant (Buckley, 2014 ; Brymer \& Schweitzer, 2017).

Noyes et ses collègues ont révisé leur modèle pour ajouter, aux côtés des processus de dépersonnalisation et des aspects mystiques de l'expérience, un facteur dit d'hyperalerte (Noyes \& Slymen, 1979). Dans leur échantillon, composé à $91 \%$ de rescapés d'accidents, de chutes et de noyades, 59\% des sujets rapportaient des éléments relatifs à cette hyperalerte: meilleure vision et audition, pensées et mouvements plus rapides et pertinents, et pensées perçues comme automatiques. Ce facteur prédominant d'hyperalerte s'appliquerait sur toute la gamme idéo-motrice de la perception pure, dans la continuité du circuit perception-pensée-motricité décrit par Bergson (1896, p. 114). Pour rester dans le cadre des troubles de l'encorporation, nous baptiserons conscience hyperincorporée le pôle vers lequel ces tendances se dirigent, et focalisation la dynamique inverse de la distanciation, qui augmente la proximité entre le sujet et son corps. Ce processus semble jouer un rôle vital dans le déroulement d'une EMI.

Dans l'échantillon de Noyes et ses collègues, ce sont ainsi $50 \%$ des 189 sujets qui relatent des efforts physiques ou mentaux extraordinaires pour échapper au danger auxquels ils étaient confrontés (Noyes \& Slymen, 1979). Cet aspect négligé est pourtant connu depuis longtemps. Heim (1892) avait lui-même recensé 30 cas, dont le sien, d'individus qui attribuaient leur sauvetage à des performances physiques et/ou intellectuelles hors norme facilitées par l'état de conscience dans lequel ils se trouvaient, état assimilable au "moi vif» ou à la conscience hyperincorporée. A l'heure actuelle, nous manquons encore de données venant confirmer la fréquence et les conditions d'occurrence de tels efforts de sauvetage, car ceux-ci semblent être sortis des radars des chercheurs. L'avantage évolutif apporté par les EMI - c'est-à-dire leur contribution à la survie de l'espèce qui en ferait un caractère favorisé par la sélection naturelle commence seulement à être envisagé (Evrard et al., soumis; Lake, 2017), mais il constitue un argument de poids en faveur d'une perspective bergsonienne.

En effet, l'EMI pourrait être considérée comme une exaltation de la mémoire tout autant qu'une exaltation de la perception. Il faut évidemment entendre la perception au sens de Bergson, c'est-à-dire non pas comme une fonction de représentation mais comme une fonction de sélection d'images. Le degré de réalité se mesure alors au degré d'utilité (Bergson, 1896, p. 68). La perception pure est toute orientée vers l'action : «L'actualité de notre perception consiste donc dans son activité, dans les mouvements qui la prolongent, et non dans sa plus grande intensité : le passé n'est qu'idée, le présent est 
idéo-moteur.» (Bergson, 1896, p.71; ses italiques) Cet alignement idéo-moteur correspond au «plus bas degré de l'esprit - l'esprit sans la mémoire » (Bergson, 1896, p. 250). Il y a alors reconnaissance d'ordre moteur par le corps, dans l'instantané, sans qu'aucun souvenir explicite n'intervienne (Bergson, 1896, p. 100-101). L'objet est alors aperçu et provoque des mouvements liés entre eux, continus, et qui sont des automatismes naissants ou accomplis. Ces «réflexes» jouent mécaniquement des souvenirs, ils imitent les schèmes sensori-moteurs auparavant appris. L'accélération du temps subjectif se combine avec une accélération motrice. La complexité fait place à la simplicité. L'interdétermination de l'action étant fonction de la capacité d'attente du corps, la réaction se fait ici sans retard et selon un déterminisme quasi immédiat.

Dans la perception pure, le monde matériel devient alors un ensemble d'images-objets qui agissent et réagissent avec d'autres images-objets, y compris notre corps ramené à son statut fondamental. Ces automatismes n'en sont pas moins « intelligents » puisqu'ils essayent, comme à l'accoutumée, de s'adapter à la situation à partir de leçons apprises. Toutefois, la place laissée à la volonté et donc à l'interdétermination de l'action est réduite, comme s'il s'agissait d'une procédure d'urgence qui court-circuitait la bureaucratie habituelle. Nous avons repris l'expression populaire d' "énergie du désespoir » pour restituer le caractère limite de ce phénomène (Evrard et al., soumis).

Bergson (1896, p. 170) a décrit le vécu de la perception pure à travers une figure extrême qu'il appelle "l'homme d'action» et qu'il oppose au rêveur. Celui-ci est caractérisé par:

« la promptitude avec laquelle il appelle au secours d'une situation donnée tous les souvenirs qui s'y rapportent ; mais c'est aussi la barrière insurmontable que rencontrent chez lui, en se présentant au seuil de la conscience, les souvenirs inutiles ou indifférents. Vivre dans le présent tout pur, répondre à une excitation par une réaction immédiate qui la prolonge, est le propre d'un animal inférieur : l'homme qui procède ainsi est un impulsif. » (Bergson, 1896, p. 170)

Toutefois, cette impulsivité de l'homme d'action peut se révéler avantageuse dans une situation critique. Lorsqu'une personne évite un accident de la route, au volant de sa voiture, grâce à des réflexes associés à l'analyse globale et instantanée des trajectoires de tous les véhicules engagés, il s'agirait déjà d'une EMI, quoi qu'en disent certains modèles actuels qui croient distinguer une différence de nature entre la réaction aiguë au danger mortel et l'EMI (Sabom, 2008 ; pour une discussion, cf. Evrard et al., soumis). Cette exaltation de la perception n'est pas apparue évidente à Bergson à une époque où cet aspect avait été négligemment écarté par Ribot (1881) et Egger (1896a). Toutefois, cette négligence a un intérêt épistémique puisque ces aspects viennent confirmer de façon indépendante l'intérêt de la lecture bergsonienne des EMI. Cela donne même un avantage à la théorie bergsonienne qui, contrairement à d'autres modèles, est en capacité de lier les différentes dimensions de cette expérience, de rassembler le "rêveur absolu » et «l'homme d'action», comme nous nous proposons de le montrer.

\subsection{Combinaisons de la conscience désincorporée et de la conscience hyperincorporée}

Poulet (1960, p. 40) s'interrogeait déjà sur la possibilité de concilier les aspects paradoxaux dégagés par Bergson. N'est-il pas possible de concevoir une pensée qui soit à la fois tendue et détendue ? Une pensée qui relâche ses liens avec le présent de l'action immédiate, mais persiste dans son rôle attentif de pensée, à la différence du rêve ?

Le processus de disjonction semble rendre possible ce fonctionnement exceptionnel. L'expérience de la mémoire pure (conscience désincorporée) et de la perception pure 
(conscience hyperincorporée) n'y seraient plus exclusives. C'est le paradoxe déjà analysé par Noyes et Kletti (1977b, p. 383) qui indiquaient cette division chez les sujets entre un soi observant et un soi participant. En regroupant les éléments présents dans les récits d'EMI, ils listaient huit caractéristiques opposables deux à deux. Ainsi, si le soi participant expérimentait des mouvements et des pensées accélérés, le soi observant était marqué par une impression de ralentissement des mouvements et des pensées. Lorsque le soi participant vivait des émotions de façon intensifiée, le soi observant ne ressentait pas d'émotions ou alors de façon atténuée, laissant place à un calme olympien. Le découpage de Noyes et Kletti, basé empiriquement sur les réponses faites aux questionnaires, ne permettait pas de relier conceptuellement ces différentes caractéristiques. Mais la théorie bergsonienne le facilite si on assimile soi participant, pris dans l'action, avec la conscience hyperincorporée; et soi observant, pris dans la contemplation, avec la conscience désincorporée.

Le point essentiel est de convenir que l'EMI n'est pas une expérience homogène mais hétérogène, puisqu'elle est mue par deux processus contradictoires. Noyes et Kletti (1977a, p. 192) identifiaient deux mécanismes différents : l'un offensif visant à écarter la menace mortelle; l'autre défensif visant une retraite pacifique. La même bipartition se retrouve chez des auteurs connus ou non de Noyes et Kletti. Le pasteur et psychanalyste Oskar Pfister (1930), à partir des données de Heim (1892) et de ses propres expériences, distinguait une résignation mortifère (assimilée à la pulsion de mort) et un maintien du désir de se sauver (assimilé à la pulsion de vie). A sa suite, un autre psychanalyste, Sandor Ferenczi (1982), appela « autoclivage narcissique » cette scission par laquelle une partie du Moi abandonne, se soumet et se résigne à une reddition totale, tandis qu'une autre cherche à préserver et à protéger la frontière du Moi. Plus récemment, le psychanalyste Jacques Roisin (2009) a parlé de «clivage de survie» en tant que mode de défense courant dans l'instant traumatique. Cependant, ces termes de "dissociation", « clivage», "scission» et « dépersonnalisation » sont trop connotés dans le champ clinique, ce qui pourrait prématurément fermer la porte à la considération de la valeur adaptative intrinsèque à ce phénomène. C'est pourquoi nous avons suggéré le terme plus neutre de « disjonction » (Le Maléfan \& Evrard, 2009) pour cette réaction combinant une tendance à « se battre » et à « fuir » simultanément.

La notion de disjonction rend compte d'une désintrication de processus habituellement intégrés. La description de la jonction entre l'esprit et le corps est au cœur de Matière et mémoire (Bergson, 1896). Bergson la présente sous un angle dynamique, c'est-à-dire dans une tension permanente selon le degré d'attention à la vie. L'EMI serait donc la manifestation concrète d'une détente, d'un relâchement voire d'une rupture dans la relation entre l'esprit et le monde. Même si le philosophe n'a conservé des EMI que l'exaltation de la mémoire, cette réaction entraîne en fait deux produits, puisque la mémoire et la perception se retrouvent mutuellement isolées. En réintroduisant la complémentarité entre l'action et la contemplation, nous ne faisons, croyons-nous, qu'appliquer le bergsonisme, doctrine d'action (Poulet, 1960, p. 34), en comblant son appréhension nécessairement lacunaire des EMI. Et le maintien, dans l'EMI, d'une attention à la vie sous une forme que nous pourrions dire exaltée, permet d'inscrire ces expériences dans le prolongement de la démonstration entreprise dans Matière et mémoire. En somme, l'EMI n'est pas qu'une vaine contemplation de dernière minute, elle reste articulée à la perception du présent et à l'exigence pratique.

En lui-même, ce dédoublement du fonctionnement psychique est déjà connu, puisqu'on peut le rapprocher de la dissociation non-pathologique. De nombreuses pratiques visant à induire un état modifié de conscience jouent sur les deux tendances à la focalisation et 
à la distanciation pour produire une disjonction de la relation entre le corps et l'esprit. C'est le cas de l'hypnose dont la procédure d'induction utilise la fixation sur un stimulus et/ou l'ouverture à tous les stimuli de l'environnement ou de l'espace fantasmagorique de la mémoire (Cardeña, 2005). Le sujet est ainsi tiré du côté de sa mémoire, explorant le plan du rêve avec plus de lucidité et de possibilités de remaniement ; pendant que son "corps» laisse prise aux effets idéo-moteurs nourris par les suggestions de l'hypnotiseur. On retrouve ce même procédé dans les deux grandes stratégies de méditation, Samadhi et Vipassana, la première employant une focalisation sélective de l'attention en éliminant les distractions, la seconde poussant à l'observation de tous les phénomènes naturels dans l'environnement et dans l'esprit, sans chercher à interagir avec eux (Schmidt, 2012). Dans ces deux cas, mais également dans d'autres pratiques de focalisation et dans les activités qui facilitent l'automatisation du comportement (comme le jogging), on recherche un détachement par rapport au registre habituel orienté vers l'action (Holroyd, 2003).

Cette disjonction se produirait spontanément lors de l'EMI et pourrait être ressentie explicitement par certains témoins (par exemple, Evrard et al., sous presse). Noyes (1981, p. 23) a esquissé une séquence durant laquelle le sujet va d'abord se focaliser sur le danger dans l'environnement et fera des efforts pour se sauver puis, lorsque les circonstances le dépassent ou qu'il abandonne ses efforts, son attention sera redirigée vers les expériences internes, dont la mémoire panoramique. Il explique ainsi pourquoi les sujets de sa cohorte dont les expériences sont chargées d'éléments mystiques sont principalement ceux qui étaient confrontés à une maladie grave et non à un accident pouvant encore être évité. Cela suggère en effet que ces aspects mystiques sont liés à " un retrait plus complet des circonstances extrêmes » (Noyes \& Slymen, 1979, p. 318). Toutefois, cette séquence passant d'abord par la conscience hyperincorporée puis par la conscience désincorporée ne rend pas justice à tous ces témoignages qui paraissent plutôt indiquer une attention stimulée simultanément par l'extérieur et par l'intérieur. Certains sujets expriment clairement leur impression d'avoir été deux êtres à la fois, ce qu'ils désignent parfois en indiquant qu'une partie d'eux-mêmes semblait contrôlée par un agent extérieur. Il arrive ainsi qu'ils ne se reconnaissent pas dans les acrobaties auxquelles ils doivent la vie et finissent par les attribuer à une intentionnalité extrahumaine. Conformément à la suggestion de Janice Holden (2009, p. 185-186), ces deux consciences doivent plutôt être considérées comme des aspects et non comme des phases de l'expérience.

Nous postulons que l'objectif de cette disjonction est de maximiser les chances de produire des "actions de survie » en mobilisant toutes les ressources de l'organisme, c'est-à-dire la conscience hyperincorporée, face à une menace mortelle perçue comme immédiate (même si celle-ci se révèle finalement être factice ; cf. Gabbard \& Twemlow, 1991). Quelle pourrait être la contribution de la conscience désincorporée ? Certains auteurs ont suggéré que la contemplation des souvenirs, dans une attitude sereine d'acceptation des événements, offrait un "refuge pour la psyché » qui réduisait les répercussions post-traumatiques (Le Maléfan, 2011), du moins sur le court terme (Roisin, 2010). D'autres que cet état d'esprit facilitait l'action en prévenant le choc émotionnel de l'effroi et la paralysie subséquente (Heim, 1892), et en faisant émerger la conviction infaillible d'une action encore possible en dépit du danger (Pfister, 1930). Il ne manque donc pas d'hypothèses psychologiques sur le possible avantage adaptatif de la conscience désincorporée.

Quoi qu'il en soit, la production première de cette conscience est ce que nous proposons d'appeler un "scénario de survie ». La conclusion de ce scénario est toujours identique 
puisque le sujet retourne à son état de conscience habituel, ce qui paraît être, de son point de vue, un « retour à la vie », et cela en dépit des péripéties vécues : même lorsqu'il franchit volontairement ou involontairement ce qui apparaissait comme un point de non-retour, même lorsqu'il choisit de rester mort ou de revenir à la vie, de répondre aux directives d'en haut ou aux appels d'en bas, le scénario prend fin car le sujet a survécu à son périple (Augustine, 2007). Parfois, la fin du scénario semble précipitée par le fait que le sujet vient d'être réanimé par l'équipe de secours. Il y a alors coïncidence parfaite entre le retour et l'extinction de la menace. Toutefois, il s'agit là d'un cas particulier d'un phénomène plus général : dans les récits des sujets, l'arrêt de l'EMI est présenté comme coïncidant avec la fin de la perception de la mort imminente, c'est-à-dire lorsque la menace est levée soit par les actions du sujet, soit par celles d'autrui, ou soit parce que la dangerosité est réévaluée. Ainsi, nous avons communiqué le cas d'un homme tombant dans une eau qu'il croyait profonde et à laquelle il ne pensait pas réchapper, avant de se rendre compte qu'il restait très facilement à la surface. Dans le très bref laps de temps de sa chute, il vécut la plupart des éléments d'une EMI (Evrard et al., sous presse). Un cas comparable est celui d'une recrue de l'armée qui fait une EMI après avoir fait tomber à terre une grenade, avant de se rendre compte, alors qu'il était sorti de son corps et entré en contact avec des entités, que la grenade n'avait pas explosé car elle était factice (Gabbard \& Twemlow, 1991).

Nous pouvons également représenter le processus de l'expérience de mort imminente en trois étapes coordonnées [voir Figure 3]:

1) Il y a disjonction des deux mémoires et perte de l'unité de la conscience, divisée en une conscience désincorporée et une conscience hyperincorporée ;

2) La conscience désincorporée, par un mouvement de distanciation, s'oriente vers un scénario de survie à partir des souvenirs personnels.

3) Dans le même temps, la conscience hyperincorporée, par un mouvement de focalisation, s'oriente vers une action de survie à partir des habitudes motrices. 
Figure 3 : Trois étapes de l'expérience de mort imminente

ETAPE 1

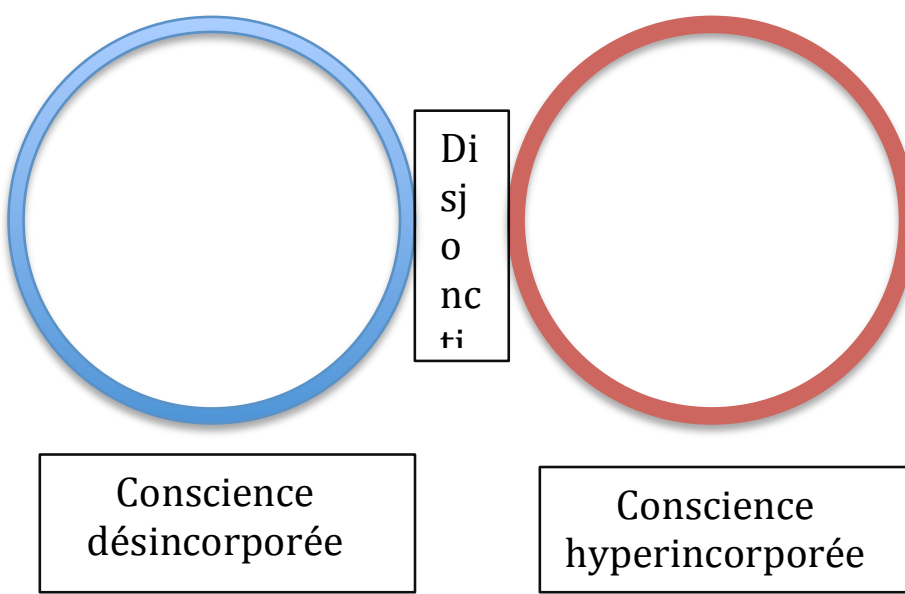

\section{ETAPES 2 ET 3}

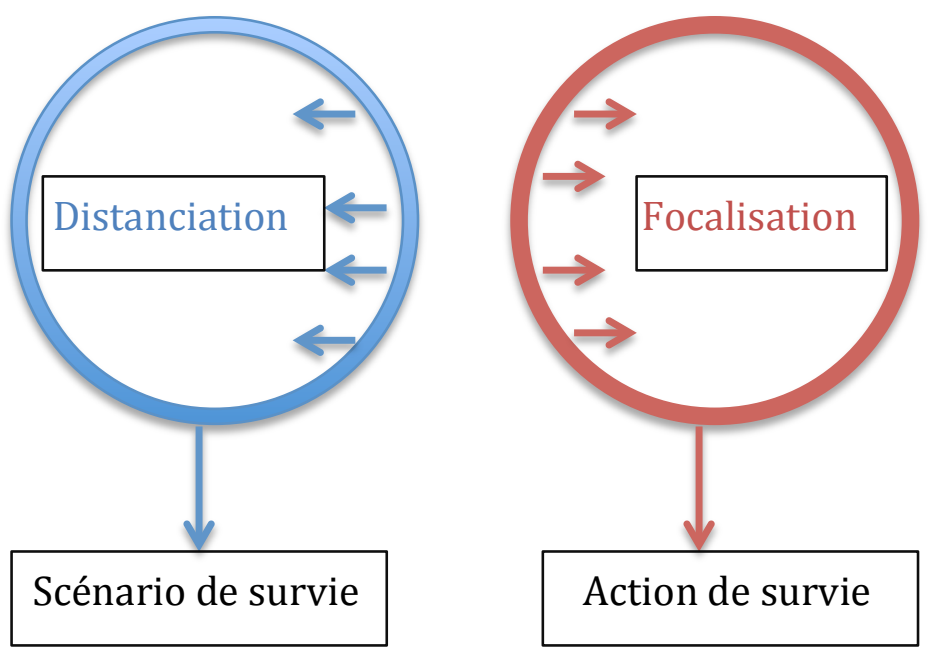


Il serait intéressant de montrer les liens entre les deux phénomènes du "scénario de survie» et de "l'action de survie», et non seulement leur simultanéité. Sont-ils véritablement indépendants jusqu'à leur résolution finale? L'un peut-il influencer l'autre, soit à partir de la base de souvenirs personnels, soit à partir de l'attitude coporelle qui va déclencher la sélection du souvenir? En somme, ce processus exceptionnel que nous appelons disjonction montre-t-il véritablement une rupture entre ces deux mémoires aux fonctionnements habituellement interpénétrés, ou laisse-t-il voir une autre forme de mixité, de collaboration? Comment se juxtaposent ces phénomènes au niveau temporel quand le scénario de survie transforme quelques secondes ou minutes en vécu d'éternité (Dron, 2009)?

L'interruption de la perception de la mort imminente est l'occasion de rassembler les parties disjointes du fonctionnement psychique. Cela n'a rien d'évident: de nombreux sujets constatent un décalage avec leur fonctionnement précédent. Le retour dans la corporalité est fréquemment décrit de façon négative (Jourdan, 2007). La psychologie du sujet peut également évoluer en entraînant parfois une désadaptation et de la nostalgie de l'EMI (Evrard, 2013). Mais des contreparties positives sont également recensées (Holden et al., 2009) : changements psychologiques à valence positive, développement de nouvelles capacités artistiques, guérisons de maladies somatiques plus ou moins sévères, impressions de vivre davantage d'expériences paranormales et d'avoir même développé des capacités parapsychiques, etc. Cette "nouvelle jonction » peut nécessiter un accompagnement thérapeutique et des dispositifs cliniques ont récemment vu le jour sur des bases scientifiquement mal assurées (Perera, Jagadheesan, Peake, 2011; Greyson, 2013).

\subsection{Perspectives de validation empirique de ce modèle}

Les notions de "conscience désincorporée » et de "conscience hyperincorporée » tentent de rendre compte, avec d'autres termes, des vécus de "mémoire pure » et de " perception pure » décrits par Bergson. Elles n'en renvoient pas moins à des hypothèses qui nécessitent davantage d'investigations avant de pouvoir prétendre prendre place dans les théories cognitives contemporaines.

Générer artificiellement une expérience de mort imminente dans le cadre d'une expérimentation ne va pas sans poser de nombreux problèmes éthiques. Si le déclencheur est bien la perception, par surprise, d'une menace mortelle pour le sujet, ce stimulus est difficile à produire sous une forme non-atténuée et contrôlée. Comment recréer ce «brusque désintéressement de la vie né de la conviction soudaine qu'on va mourir à l'instant » (Bergson, 1919, p. 77) ? Le sujet installé en laboratoire ou dans des conditions naturelles devra donner au préalable son consentement pour participer à l'expérience et sera donc dans une attitude d'anticipation et de relativisation des stimuli menaçants présentés par l'expérimentateur. De plus, les importantes répercussions positives comme négatives de ces expériences font qu'elles ne peuvent pas être administrées à la légère. Toutefois, des chercheurs ont le projet de provoquer des expériences qui se rapprochent de l'EMI en employant différents moyens : l'hypnose, la réalité virtuelle, la syncope et des drogues psychotropiques (en particulier, la kétamine, cf. Le Maléfan, 2017; Smythies, 2011) ont été proposées pour induire la phénoménologie des EMI tout en la couplant avec une imagerie cérébrale de haute résolution (Charland-Verville, 2016, p. 72-73).

En attendant que ces méthodes aient fait leurs preuves et puissent s'accorder avec une pratique de recherche valide sur le plan éthique, il semble donc nécessaire de chercher à 
vérifier ce modèle à partir d'une analyse des cas spontanés, ce qui n'empêche guère une étude prospective avec des personnes médicalisées qui seraient amenées à faire une EMI. Une meilleure compréhension de ce qui s'y joue pourrait permettre de provoquer des expériences équivalentes dans des conditions contrôlées et avec toutes les garanties nécessaires.

Notre modèle bergsonien des EMI pourrait se vérifier en explorant sur une cohorte de taille importante ces aspects auparavant négligés :

- Le sujet perçoit-il une disjonction entre deux consciences ayant des visées et des moyens différents? Peut-on dégager ces deux consciences par une analyse factorielle de son récit?

- Ressent-il une distorsion de la perception du temps associée à la fois à l'accélération de sa vie psychique et à l'enrichissement de sa perception du monde extérieur?

- Expérimente-t-il des automatismes psychomoteurs? Ou ceux-ci sont-ils attestés par des témoins de l'expérience ou d'autres éléments de preuve?

- Le sujet réalise-t-il des performances physiques ou intellectuelles hors du commun? Celles-ci ont-elles véritablement contribué, en temps réel, à sa survie?

Ces éléments devront être mis en parallèle avec les conditions de survenue de l'expérience afin de vérifier la connexion entre la forme de l'expérience et la perception de la menace vitale. Nous pourrons ainsi confirmer ou infirmer la séquence décrite par Noyes et Kletti (1976b, p. 108) :

"Aussi longtemps qu'une possibilité de s'en sortir demeure, une énorme énergie peut être consacrée à des actions de sauvetage. La phase de résistance cesse lorsque l'individu, reconnaissant la futilité de sa lutte, s'abandonne à la mort. Ce moment de capitulation est accompagné de façon caractéristique par un sentiment de profonde tranquillité, et même de joie. »

Ainsi, dans tous les cas où un sauvetage semble possible, du point de vue du sujet, celuici passerait par une phase de résistance dominée par la conscience hyperincorporée. Puis, s'il abandonne ou est contraint de capituler, ou dans les cas où il perçoit d'emblée que tout sauvetage est impossible, il devrait passer aux autres phases dites de « revue de vie » et de «transcendance» (Noyes \& Kletti, 1976b) dominées par la conscience désincorporée.

La vérification de ce modèle passe également par la mise en évidence d'un continuum entre les expériences aiguës de confrontation avec le réel de la mort (Le Maléfan \& Coq, 2015 ; Roisin, 2010 ; Sabom, 2008) et les EMI dans lesquelles les éléments mystiques sont prédominants (Moody, 1975). Les expériences le long de ce continuum pourraient ne pas avoir les mêmes effets dans l'après-coup. L'intensité et la durée de la disjonction entre conscience désincorporée et hyperincorporée expliqueraient cette variabilité des EMI et de leurs conséquences, attribuée aujourd'hui à la notion vague de "profondeur » (Colombel, 2003). Plutôt que de distinguer les EMI sur une base purement quantitative, c'est-à-dire en fonction du nombre d'items endossés par le sujet (Khanna \& Greyson, 2014), alors que ces items ne sont pas indépendants les uns des autres, il serait nécessaire de reconstruire une échelle plus valide en se basant sur la reconstruction phénoménologique que nous proposons. Ainsi, les items relatifs à la conscience désincorporée et au scénario de survie formeraient un ensemble cohérent, et d'autres items rejoindraient la catégorie de la conscience hyperincorporée et des actions de survie. Cette échelle impliquerait de repenser la phénoménologie des EMI, qui est actuellement loin du consensus, en prenant en compte l'hétérogénéité qui y est à l'œuvre. En effet, pour prendre un exemple, le temps sera vécu à la fois comme accéléré 
et ralenti, puisque le sujet baigne simultanément dans une perspective contemplative et une perspective orientée vers l'action (Wittman et al., sous presse). Une telle distinction est noyée dans l'échelle de Greyson $(1983,1990)$ où la première question à laquelle le sujet doit répondre est : « Le temps semblait-il s'accélérer ou ralentir ? »6

\section{Conclusion}

Bergson avait considéré que l'exaltation de la mémoire survenant dans les EMI était à la fois un phénomène digne de considération et des plus instructifs pour la philosophie. Réciproquement, le modèle des deux mémoires de Bergson offre un cadre de compréhension unique pour accueillir ces expériences. Sous sa plume, elles apparaissent, dans un premier temps, comme la conséquence d'une levée d'inhibition, faisant suite à un désintéressement pour l'action efficace, qui lui-même trouverait sa cause dans un déséquilibre sensori-moteur. Puis, dans un second temps, Bergson perçoit qu'un déclencheur psychologique (la conviction soudaine de la mort propre) peut tout à fait entraîner ce déséquilibre sensori-moteur. Il réévalue alors l'importance de ce déclencheur qui oppose symboliquement vie et mort. Il ne s'agit donc plus d'un simple affaiblissement de l'attention pour la vie, mais d'un relâchement de l'attention de l'espèce, en d'autres termes, d'une rupture de l'élan vital. Ainsi, Bergson lie autour de ce phénomène plusieurs pans de son œuvre théorique.

Toutefois, il ne va pas plus loin dans son investigation et son analyse du phénomène. C'est avec des données plus complètes issues d'un siècle de recherche que l'on parvient à saisir à quel point le modèle de Bergson rend intelligibles, avec un faible nombre de propositions, la mémoire panoramique et les autres anomalies par lesquelles l'esprit semble spontanément prendre ses distances avec le fonctionnement corporel normal. Mais ce versant contemplatif ne devient cohérent qu'en prenant en compte le versant actif : l'EMI est d'abord un état modifié de conscience qui semble favoriser des actions de sauvetage assurant la survie du sujet. Réintroduire ce versant est évident car le bergsonisme "demeure, en dépit de tout, une doctrine d'action, et non d'inaction et d'abandon» (Poulet, 1960, p.34). Cependant, cette explication est limitée car elle s'applique aux EMI survenant dans des contextes traumatiques de perception d'une menace mortelle ; or le même phénomène survient, selon toute apparence, dans des conditions où aucune menace objective n'est identifiée a posteriori (Charland-Verville et al., 2014). Il faudrait alors soit développer une hypothèse auxiliaire selon laquelle le même processus de disjonction pourrait être actionné par différents biais ; soit retenir la primauté de la perspective subjective quant à l'évaluation de la menace. Ainsi, un sujet en pleine méditation peut sentir qu'il entre dans un désintéressement de la vie qui l'amène à se distancer de son corps et du présent, ce qui est d'ailleurs parfois le but recherché. Toutefois, sur le plan phénoménologique, il n'y a pas de différences significatives entre les récits des «vraies EMI » et des «simili-EMI ( NDE-like, selon la terminologie en vigueur) qui justifieraient de distinguer ces catégories sur une base uniquement étiologique (Owens et al., 1990 ; Charland-Verville et al., 2014).

\footnotetext{
6 Dans la version originale de l'échelle (Greyson, 1983), l'item 1 demandait simplement « Est-ce que le temps semble s'accélérer? » ayant comme possibilités de réponse : «1) Non. 2) Le temps semble aller plus vite que d'habitude. 3) Tout semble se produire en même temps. ». Cet item a été modifié dans une version révisée de l'échelle (Greyson, 1990) du fait du problème de perspective prise par le sujet pour évaluer l'écoulement du temps. Les trois nouvelles options sont depuis : «1) Non. 2) Le temps semble aller plus vite ou moins vite que d'habitude. 3) Tout semble se produire en même temps; ou le temps s'est arrêté, ou il a perdu toute signification. » Le même item peut donc correspondre à des vécus très différents.
} 
Si Bergson discute, avec d'autres chercheurs, de la connexion entre sa théorie d'une mémoire pure et la survie de quelque chose à la disparition de notre corps physique, sa théorie nous permet de maintenir l'EMI parmi les phénomènes psychologiques ayant d'abord une visée utilitariste pour le vivant. L'EMI ne concernerait la mort qu'en ce qu'elle peut d'abord permettre de l'éviter, au prix d'une rupture temporaire et partielle de la jonction entre l'esprit et le corps, qui ne va pas sans risques. Dans l'après-coup de cette réaction psychosomatique ultime, certains sujets semblent devoir se réadapter à leur corps et réaménager leur fonctionnement psychique pour intégrer ce vécu hyperréaliste et presque indélébile. Leur tâche n'en est que plus difficile du fait des modèles interprétatifs qui dominent aujourd'hui, entre ceux qui cherchent à réduire ce vécu à un raté cérébral et ceux qui veulent le tirer vers une spiritualité anti-matérialiste. Le dualisme interactionniste de Bergson n'est probablement pas la solution scientifique actuellement la plus prisée, mais elle propose un cadre fécond pour penser ces vécus au sein d'une théorie globale et cohérente des relations entre l'esprit et le corps.

\section{Références}

Alvarado, C.S. (2000). Out-of-body Experiences. In: E. Cardeña, S.J. Lynn, \& S. Krippner (Eds.), Varieties of anomalous experiences (pp. 183-218). Washington: American Psychological Association.

Alvarado, C.S. (2011). Panoramic Memory, Affect, and Sensations of Detachment in the Dying: Discussions Published in France, 1889-1903. Journal of Near-Death Studies, $30(2), 65-82$.

Arstila, V. (2012). Time slows down during accidents. Frontiers in Psychology, 3(196), 1-10. doi: 10.3389/fpsyg.2012.00196

Augustine, K. (2007). Hallucinatory Near-Death Experiences. Journal of Near-Death Studies, 26, 3-31.

Bergson, H. (1886). De la simulation inconsciente dans l'état d'hypnotisme. Revue philosophique de la France et de l'Etranger, 22, 525-531.

Bergson, H. (1889). Essai sur les données immédiates de la conscience. Paris : Alcan.

Bergson, H. (1901). Le rêve. Bulletin de l’institut général psychologique, 1(3), 103-122.

Bergson, H. (1907). L'évolution créatrice. Paris : Alcan.

Bergson, H. (1913). Discours prononcé le 28 mai 1913, en prenant possession du siège présidentiel de la Society for Psychical Research de Londres. Annales des sciences psychiques, 23(11-12), 321-328.

Bergson, H. (1914-1915). Presidential address. Proceedings of the Society for Psychical Research, 27, 157-175.

Bergson, H. (1919). L'énergie spirituelle. Paris : Alcan.

Bergson, H. (1922). Durée et simultanéité. A propos de la théorie d'Einstein. Paris : Alcan.

Bergson, H. (1932). Les deux sources de la morale et de la religion. Paris : Alcan.

Bergson, H. (1934). La pensée et le mouvant. Paris : Alcan.

Bergson, H. (1959). Matière et mémoire: Essai sur la relation du corps à l'esprit [1896]. Paris: PUF.

Brymer, E., Schweitzer, R. (2017). Evoking the ineffable: The phenomenology of extreme sports. Psychology of Consciousness: Theory, Research, and Practice, 4(1), 63-74.

Buckley, R. (2014). Slow time perception can be learned. Frontiers in Psychology, 5(209). doi:10.3389/fpsyg.2014.00209 
Cannon, W.B. (1915). Bodily changes in pain, hunger, fear and rage. An account of recent researches into the function of emotional excitement. New York \& London: D. Appleton \& Cie.

Capek, M. (1971). Bergson and modern physics. A reinterpretation and re-evalution. Dordrecht: Reidel Publishing Company.

Cardeña, E. (2005). The phenomenology of deep hypnosis: Quiescent and physically active. International Journal of Clinical and Experimental Hypnosis, 53, 37-59.

Charland-Verville, V. (2016). The exploration of the content of memories after coma. Thèse présentée en vue de l'obtention du grade de Docteur en Sciences Médicales, Université de Liège.

Charland-Verville, V., Jourdan, J.-P., Thonnard, M., Ledoux, D., Donneau, A.-F., Quertemont, E., Laureys, S. (2014). Near-death experiences in non-life-threatening events and coma of different etiologies. Frontiers of Human Neurosciences, 8, 203. doi: $10.3389 /$ fnhum. 2014.00203

Colombel, J.-C. (2003). La profondeur des N.D.E., entre mythes et logos. Les cahiers $d u$ réseau Psychologie et réanimation, $\mathrm{n}^{\circ} 19,63-73$.

Coppes, C. (2013). The Essence of Religions: A Glimpse of Heaven in the Near-Death Experience. New York : Select Books Inc.

Corman, M., Monier, F., Sicard, A., Da Fonseca, A., Didelot, T., Hallez, Q., Kchani, A., Salomone, M., Vichy, G., Dambrun, M. (2017). L'Expérience de mort imminente (EMI) : une synthèse de la littérature. L'Année psychologique / Topics in Cognitive Psychology, 1-25.

Csíkszentmihályi, M. (1990). Flow: The Psychology of Optimal Experience. New York: Harper and Row.

Deleuze, G. (1966). Le Bergsonisme. Paris : PUF.

Dron, N. (2009). 45 secondes d'éternité : mes souvenirs de l'au-delà. Paris : Kymzo.

Dunne, B., Jahn, R.G., Hoeger, E., Jones, Z. (2009, eds.). Filters and Reflections. Perspectives on Reality. New York : ICRL Press.

Egger, V. (1895). La durée apparente des rêves. Revue Philosophique de la France et de l'étranger, 20, 41-59.

Egger, V. (1896a). Le moi des mourants. Revue philosophique de la France et de l'étranger, $21,26-38$.

Egger, V. (1896b). Le moi des mourants - faits nouveaux. Revue philosophique de la France et de l'étranger, 21, 337-368.

Evrard, R. (2013). Répercussions psychologiques des « souvenirs » de la mort propre: une critique des travaux du Dr Pim van Lommel. Etudes sur la mort, n ${ }^{\circ} 143,159-172$.

Evrard, R. (2016). La légende de l'esprit : enquête sur 150 ans de parapsychologie. Paris : Trajectoire.

Evrard, R., Lazrak, N., Laurent, M., Toutain, C., Le Maléfan, P. (sous presse). Du « moi vif» des noyés à l'expérience de mort imminente : approche clinique d'une énigme médicopsychologique à partir d'un nouveau cas. Annales médico-psychologiques.

Evrard, R., Toutain, C., Glazier, J.W., Le Maléfan, P. (soumis). The energy of despair: do near-death experiences have a evolutionary value? Psychology of Consciousness.

Ferenczi, S. (1982). Traumatisme et aspiration à la guérison [1930]. In: Euvres complètes IV (1927-1933) (pp. 278-280). Paris: Payot.

Fracasso, C., Friedman, H. (2011). Near-Death Experiences and the Possibility of Disembodied Consciousness. Challenges to Prevailing Neurobiological and Psychosocial Theories. NeuroQuantology, 9(1), 41-53.

Gabbard, G.O., \& Twemlow, S.W. (1991). Do "near death experiences" occur only neardeath?-Revisited. Journal of Near-Death Studies, 10, 41-47. 
Gallois, P., Forzy, G. (1997, dir.). Bergson et les neurosciences. Paris : Institut synthélabo pour le progrès de la connaissance.

Greyson, B. (1983). The near-death experience scale: Construction, reliability, and validity. Journal of Nervous and Mental Disease, 171, 369-375.

Greyson, B. (1990). Near-Death Encounters With and Without Near-Death Experiences: Comparative NDE Scale Profiles. Journal of Near-Death Studies, 8(3), 151-161.

Greyson, B. (2013). Getting comfortable with near-death experiences. Missouri Medicine, 110(6), 471-477.

Haddock, J.W. (1851). Somnolism \& Psycheism (second edition). London: James S. Hodson.

Heim, A. (1892). Notizen über den Tod durch Absturz. Jahrbuch des Schweizer Alpenclub, 27, 327-337.

Holden, J.M. (2009). Veridical Perception in Near-Death Experiences. In : J.M. Holden, B. Greyson, \& D. James, (Eds.), The Handbook of Near-Death Experiences. Thirty years of investigation (pp. 185-211). Santa Barbara, CA: Praeger.

Holden, J.M., Greyson, B., \& James, D. (2009, eds.). The handbook of near-death experiences. Thirty years of investigation. Santa Barbara, CA: Prager/ABC-CLIO.

Holroyd, J. (2003). The science of meditation and the state of hypnosis. American Journal of Clinical Hypnosis, 46, 109-128.

Irwin, H.J. (2000). The Disembodied Self: An Empirical Study of Dissociation and the Outof-Body Experience. Journal of Parapsychology, 64, 261-277.

Jourdan, J.-P. (2007). Deadline: dernière limite. Paris: Les 3 orangers.

Kastrup, B. (2012). A paradigm-breaking hypothesis for solving the mind-body problem. Paranthropology, 3(3), 4-12.

Katz, J., Saadon-Grosman, N., Arzy, S. (2017). The life review experience: Qualitative and quantitative characteristics. Consciousness and Cognition, 48, 76-86.

Kelly, E.F., Kelly, E.W., Crabtree, A., Gauld, A., Grosso, M., \& Greyson, B. (2007). Irreducible Mind: Toward a Psychology for the $21^{\text {st }}$ Century. Lanham, MD: Rowman \& Littlefield.

Khanna, S., Greyson, B. (2014). Near-death experiences and spiritual well-being. Journal of Religion and Health, 53(6), 1605-1615.

Lake, J. (2017). The evolution of a predisposition for the near-death experience: implications for non-local consciousness. Journal of Nonlocality, 5(1), 1-33.

Laureys, S. (2015). La lumière au bout du tunnel : les expériences de mort imminente. In : Un si brillant cerveau. Les états limites de conscience (pp. 139-151). Paris : Odile Jacob.

Lenay, C. (2006). Enaction, externalisme et suppléance perceptive. Intellectica, n43, 27-52.

La Harpe, J. de (1941). Souvenirs personnels d'un entretien avec Bergson. In: A. Béguin \& P. Thévenaz (dir.), Henri Bergson. Essais et témoignages inédits (pp. 357-364). Paris : La Baconnière.

Le Maléfan P. Vécu de mort imminente et onirisme. Un chapitre inattendu de l'histoire de la psychologie dynamique. L'Information psychiatrique $1995 ; 8: 773-80$.

Le Maléfan, P. (1995). Vécu de mort imminente et onirisme. Un chapitre inattendu de l'histoire de la psychologie dynamique. L'Information psychiatrique, 8, 773-780.

Le Maléfan, P. (2011). Le joint le plus intime du sentiment de la vie. La rencontre traumatique, de Heim à Ferenczi, avec Freud et Lacan. Revue Francophone du Stress et du Trauma, 11(4), 197-203.

Le Maléfan, P. (2017). Être décorporé sous kétamine : clinique et coïncidences. Cliniques méditerranéennes, $\mathrm{n}^{\circ} 96,205-217$.

Le Maléfan, P., \& Coq, J.-M. (2015). L'instant du traumatisme. Annales médicopsychologiques, 173(2), 180-185.

Le Maléfan, P., \& Evrard, R. (2009). Comments on two Anglo-Saxon theories of the "exit 
outside the body". In: C.D. Murray (Ed.), Psychological Scientific Perspectives on Out of Body and Near Death Experiences (pp. 71-72). New York: Nova Science Publishers.

Martial, C., Charland-Verville, V., Dehon, H., Laureys, S. (2017). False memory susceptibility in coma survivors with and without a near-death experience. Psychological research. DOI 10.1007/s00426-017-0855-9

Martial, C., Cassol, H., Antonopoulos, G., Charlier, T., Heros, J., Donneau, A.-F., CharlandVerville, V., \& Laureys, S. (2017). Temporality of Features in Near-Death Experience Narratives. Frontiers in Human Neurosciences. 11:311. doi: 10.3389/fnhum.2017.00311

Moody, R. (1975). Life after Life. Atlanta, GA: Mockingbird Books.

Moore, L.E., Greyson, B. (2017). Characteristics of memories for near-death experiences. Consciousness and Cognition, 51, 116-124.

Murphy, M., White, R.A. (1995). In the Zone: Transcendent Experience in Sports. New York: Penguin Books.

Noyes, R. (1981). The Encounter with Life-Threatening Danger: Its Nature and Impact. Essence, 5, 21-32.

Noyes, R., \& Kletti, R. (1976a). Depersonalization in the face of life-threatening danger: A description. Psychiatry, 39, 19-27.

Noyes, R., \& Kletti, R. (1976b). Depersonalization in the face of life-threatening danger: An Interpretation. Omega, 7, 103-114.

Noyes, R., \& Kletti, R. (1977a). Panoramic Memory: A Response to the Threat of Death. Omega, 8, 181-194.

Noyes, R., \& Kletti, R. (1977b). Depersonalization in response to life-threatening danger. Comprehensive Psychiatry, 18, 375-384.

Noyes, R., \& Slymen, D. (1979). The subjective response to life-threatening danger. Omega, 9, 313-321.

Owens, J.E., Cook, E.W., Stevenson, I. (1990). Features of "near-death experience" in relation to whether or not patients were near death. Lancet, 336, 1175-1177.

Palmieri, A., Calvo, V., Kleinbub, J. R., Meconi, F., Marangoni,M., Barilaro, P., Broggio, A., Sambin, M., \& Sessa, P. (2014). "Reality" of near-death-experience memories: evidence from a psychodynamic and electrophysiological integrated study. Frontiers in Human Neuroscience, 8, 429.

Papanicolaou, A.C., Gunter, P.A.Y. (1987). Bergson and modern thought. Towards a unified science. Chur: Harwood Academic Publishers.

Parnia, S. (2017). Understanding the cognitive experience of death and the near-death experience. $Q J M, 110(2), 67-69$.

Perera, M., Jagadheesan, K., Peake, A. (2011, eds.). Making Sense of Near-Death Experiences: A Handbook for Clinicians. New York : Jessica Kingsley Publishers.

Pfister, O. (1930). Schockdenken und Schockphantasien bei höchster Lebensgefahr. Internationale Zeitschrift für Psychoanalyse, 16, 430-455.

Poulet, G. (1960). Bergson et le thème de la vision panoramique des mourants. Revue de théologie et de philosophie, 10(1), 23-41.

Radvansky, G.A., Krawietz, S.A., Tamplin, A.K. (2011). Walking through doorways cause forgetting: Further explorations. The Quarterly Journal of Experimental Psychology, 64(8), 1632-1645.

Ribot, T. (1881). Les maladies de la mémoire. Paris : Germer Baillière.

Ring, K. (1984). Heading toward omega: In search of the meaning of the near-death experience. New York: Morrow.

Ring, K., Cooper, S. (1999). Mindsight. Near-Death and Out-of-Body Experiences in the Blind. Palo Alto, CA: William James Center/Institute of Transpersonal Studies. 
Roisin, J. (2009). La sortie du corps et autres expériences extrêmes en situation de traumatisme. Revue francophone du stress et du trauma, 9(2), 71-79.

Roisin, J. (2010). De la survivance à la vie. Essai sur le traumatisme psychique et sa guérison. Paris : PUF.

Rousseau, D. (2011). Near-Death Experiences and the Mind-Body Relationship: A SystemsTheoretical Perspective. Journal of Near-Death Studies, 29(3), 399-435.

Sabom, M. (1982). Recollections of death: A medical investigation. New York: Harper \& Row.

Sabom, M. (2008). The Acute Dying Experience. Journal of Near-Death Studies, 26(3), 181218.

Schmidt, S. (2012). What meditation can do: mental health and exceptional experiences. In: C. Simmonds-Moore (Ed.), Exceptional Experience and Health. Essays on Mind, Body and Human Potential (pp. 113-130). Jefferson, N.C.: McFarland.

Schwaninger, J., Eisenberg, P.R., Schechtman, K.B., Weiss, A.N. (2002). A prospective analysis of near-death experiences in cardiac arrest patients. Journal of Near-Death Studies, 20, 215-232.

Smythies, J.R. (2011). Ketamine, Bergson and NDEs. Journal of the Society for Psychical Research, 75, 148-150.

Sollier, P. (1896). L'état mental des mourants. Revue philosophique de la France et de l'étranger, 21, 303-307.

Taine, H. (1870). De l'intelligence. Paris : Hachette.

Thonnard, M., Charland-Verville, V., Brédart, S., Dehon, H., Ledoux, D., Laureys, S., \& Vanhaudenhuyse, A. (2013). Characteristics of Near-Death Experiences Memories as Compared to Real and Imagined Events Memories. PLoS ONE, 8(3), e57620. doi:10.1371/journal.pone.0057620

van Lommel, P., van Wees, R., Meyers, V., \& Elfferich, I. (2001). Near-death experience in survivors of cardiac arrest: A prospective study in the Netherlands. Lancet, 358, 20392045 .

Wittman, M., Neumaier, L., Evrard, R., Weibel, R., Schmied-Knittel, I. (soumis). Subjective time distortion during near-death experiences: an analysis of reports. Zeitschrift für Anomalistik.

Zingrone, N.L., \& Alvarado, C.S. (2009). Pleasurable Western adult near-death experiences: Features, circumstances, and incidence. In J.M. Holden, B. Greyson, \& D. James (Eds.), The handbook of near-death experiences: Thirty years of investigation (pp. 17-40). Santa Barbara, CA: Praeger/ABC-Clio. 